\title{
The Vertical Structure and Ultraviolet Spectrum of X-ray Irradiated Accretion Disks in Active Galactic Nuclei
}

\author{
Mark W. Sincell \\ Department of Physics MC 704 \\ The University of Illinois at Urbana-Champaign \\ 1110 W. Green Street \\ Urbana, IL 61801-3080 \\ Julian H. Krolik \\ Department of Physics \& Astronomy \\ The Johns Hopkins University \\ Baltimore, MD 21218
}

\begin{abstract}
Motivated by recent work indicating that the UV continuum in AGN may be produced by reradiation of energy absorbed from X-rays irradiating an accretion disk, we present a calculation of the vertical structures and ultraviolet spectra of X-ray irradiated accretion disks around massive non-rotating black holes. After finding the radial dependence of vertically-integrated quantities for these disks, we solve the equations of hydrostatic equilibrium, energy balance, and frequency-dependent radiation transfer as functions of altitude. To solve the last set of equations, we use a variable Eddington factor method. We include electron scattering, free-free, and HI, HeI, and HeII bound-free opacities and the corresponding continuum cooling processes.

The incident X-ray flux heats a thin layer of material 3-4 scale heights above the midplane of the disk. This X-ray heated skin has two layers: a radiation pressure supported region in which the UV flux is created, and, immediately above this layer, a warmer zone, optically thin to UV radiation, formed where the X-ray ionization parameter is large. In the lower layer the gas pressure is nearly independent of altitude but the temperature increases upward.

The fraction of the incident hard X-ray flux which emerges in the UV falls with increasing $\dot{m}$ (the accretion rate in Eddington units). At frequencies below the Lyman edge the slope of the continuum $\left(d \ln L_{\nu} / d \ln \nu\right)$ varies from -1.6 to 0.8 as $\dot{m} / m_{8}$ increases from 0.001 to 1 . Here $m_{8}$ is the mass of the central black hole in units of $10^{8} M_{\odot}$. In all cases examined $(0.003 \leq \dot{m} \leq 0.3$ and $\left.0.27 \leq m_{8} \leq 27\right)$, the Lyman edge appears in emission. The amplitude of the
\end{abstract}


Lyman edge feature increases with $m_{8}$ but is relatively independent of $\dot{m}$. The amplitude of the Lyman edge emission feature increases with disk inclination. Compton scattering in disk coronae can smooth the Lyman edge feature only if $\tau_{c} \gtrsim 0.5$, where $\tau_{c}$ is the Thomson depth of the coronae.

While the overall spectral shape predicted by X-ray irradiation may be compatible with observations, the Lyman edge emission feature it predicts is not. This finding raises questions for many otherwise plausible models in which $\mathrm{X}$-ray irradiation plays a major role.

\section{Introduction}

The optical and UV emission of radio-quiet active galactic nuclei (AGNs) is dominated by a quasi-thermal component, the "Big Blue Bump" (Shields 1978, Malkan \& Sargent 1982, Malkan 1983). The Big Blue Bump is usually interpreted as thermal emission from a geometrically thin, optically thick, accretion disk around a massive black hole (Malkan 1983, Sun \& Malkan 1989, Laor \& Netzer 1989: LN89). This model makes two key predictions. First, the large jump in the absorptive opacity at the Lyman edge combined with the vertical temperature gradients in the disk insure that some feature will be present at the Lyman edge (Kolykhalov \& Sunyaev 1984, LN89). For the same reason, some have predicted that the polarization of the disk should decrease at frequencies above the Lyman edge (e.g., Laor, Netzer \& Piran 1990; but see Blaes \& Agol 1996 for another view). Second, variations in different portions of the optical/ultraviolet band can be associated with fluctuations in the conditions at particular radii. If fluctuations move radially through the disk, there should be time-delays between variations seen at different wavelengths which correspond to signal travel times within the disk.

Unfortunately, while this picture is very attractive, it suffers from severe problems, both theoretical and observational. From the theoretical point of view, it is generally expected that the viscous stress is proportional to the pressure (the " $\alpha$-model" introduced by Shakura \& Sunyaev 1973: SS73), including the radiation pressure when the disk is optically thick. Unfortunately, if this is true, when the accretion rate is more than a small fraction of the Eddington rate, the disk is thermally unstable throughout its inner region (Shakura \& Sunyaev 1976). For this reason, the most detailed disk spectral calculations made to date (LN89) supposed that the stress was instead proportional to the geometric mean of the gas and radiation pressures.

In addition, neither of the two key predictions just described is confirmed by 
observations. Partial Lyman edges have been detected in very few AGN (Antonucci, Kinney \& Ford 1989; Koratkar, Kinney \& Bohlin 1992) and the polarization of two quasars with partial absorption edges rises across the Lyman edge (Koratkar, et al. 1995). Intensive monitoring of the type 1 Seyfert galaxy NGC 5548 revealed that variations from the optical to the far-UV are tightly correlated. The signal travel speed through a conventional disk that would be required to coordinate these variations was found to be at least $\sim 0.1 c$, far higher than any of the expected signal speeds (Krolik et al. 1991). Moreover, variations in the soft X-ray flux have been found to be almost as tightly correlated with ultraviolet fluctuations (Clavel, et al. 1992). These two findings suggest that the Big Blue Bump may actually be due to reprocessed X-ray emission (e.g., Rokaki, Collin-Souffrin, \& Magnan 1993).

Other evidence also points to the importance of X-ray reprocessing in the formation of AGN continua. In many type 1 Seyfert galaxies, there is a spectral feature in the vicinity of $10-30 \mathrm{keV}$ which is readily interpreted as "Compton reflection" of X-rays from a cool, optically thick surface occupying roughly half of the solid angle around the X-ray source (Pounds et al. 1990). It would be very natural to interpret this reflector as an accretion disk (Lightman \& White 1988) (but a number of other interpretations are also possible: Nandra \& George 1994; Krolik, Madau, \& Życki 1994; Ghisellini, Haardt \& Matt 1994). Further support for the thought that the disk is subjected to intense X-ray irradiation comes from the discovery that the Fe K $\alpha$ profiles in many AGN are extremely broad (e.g., as summarized by Nandra 1997).

Recently popular models for the production of the X-rays are also consistent with this picture in which a large fraction of the emitted X-rays strike a nearby cool surface, possibly the accretion disk (Haardt \& Maraschi 1993: HM93; Haardt, Maraschi \& Ghisellini 1994; Pietrini \& Krolik 1995; Stern et al. 1995). These models suppose that a large part of the disk's dissipation takes place in a small amount of mass at or above the disk's surface. The very large heating rate per unit mass in an optically thin gas creates a very hot corona which cools by inverse Compton scattering. Roughly half the X-ray flux generated strikes the disk, and is reprocessed into UV photons which then return to the corona as seeds for inverse Compton scattering. Fits to the broad-band X-ray spectra of AGN are at least qualitatively consistent with this sort of thermal Comptonization model (e.g. Zdziarski et al. 1995).

In its simplest form, the corona model predicts that the observed UV flux should be roughly equal to the observed intrinsic X-ray flux (i.e. the X-ray flux after subtracting any components due to reprocessing). Whether this prediction is consistent with the observations is uncertain. The UV/X-ray flux ratio is conventionally parameterized by $\alpha_{o x}$, 
the spectral index of a power-law interpolated between $2500 \AA$ and $2 \mathrm{keV}$, as measured in the AGN rest-frame. Depending on the sample definition, the observed distribution of $\alpha_{o x}$ varies somewhat, but typically it ranges from $\simeq 1.2$ to $\simeq 2.1$, with a tendency for lower luminosity AGN to have smaller $\alpha_{o x}$ (Stocke et al. 1991; Wilkes et al. 1994; Green et al. 1995). The flux at at $2500 \AA$ is $\simeq 20 \times 400^{\alpha_{o x}-1.5}$ times the flux at $2 \mathrm{keV}$ so that, if we compare at these two frequencies alone, we would conclude that the UV luminosity exceeds the X-ray luminosity in most AGN. However, to find the ratio of the total UV and X-ray fluxes, substantial - and very uncertain-bolometric corrections must be applied to both. We cannot observe low-redshift AGN in the rest-frame EUV, but composite high-redshift quasar spectra (e.g. Zheng et al. 1996) suggest that the spectrum rolls over sharply at wavelengths shorter than $\simeq 1000 \AA$. If this shape is general, the UV bolometric correction (relative to $\nu F_{\nu}$ at $2500 \AA$ ) might be as small as $\simeq 2$, but there is likely to be a considerable dispersion from case to case. The X-ray bolometric correction is similarly uncertain. Those low luminosity AGN for which OSSE has been able to obtain spectra typically show power-laws with indices $\simeq 0.9$ across the hard X-ray range, supplemented by a substantial bump at a few tens of keV which is generally believed to be due to reprocessing (Zdziarski et al. 1995). If that power-law extends from 0.5 to $200 \mathrm{keV}$, the bolometric correction relative to $\nu F_{\nu}$ at $2 \mathrm{keV}$ is 6.5 . Taking these bolometric corrections at face value, we would conclude that the UV and X-ray fluxes in low luminosity AGN (where the evidence for the corona model is best) are indeed close to being equal, while the UV flux is typically rather greater than the intrinsic X-ray flux in higher luminosity AGN. However, it is clear from the character of this estimate that it is very weakly based. The situation is made still cloudier by the possibility that relativistic effects may direct the majority of the emitted X-rays toward the disk, reducing the observed ratio between X-rays and UV (Martocchia and Matt 1996).

It is our goal to begin the process of testing these coronal models by comparing the ultraviolet spectrum they predict with observations. In this paper we develop the techniques necessary to compute the predicted UV spectra, and apply them to the simplest version of the model: that in which all the dissipation takes place in a slab-like corona resting directly on top of the accretion disk. In future work we will examine other variations, such as models in which the dissipation is shared between the disk proper and the corona, and models with more complicated coronal geometry.

An immediate consequence of this picture in which most of the dissipation takes place in a corona, and not inside the disk proper, is that radiation pressure support in the bulk of the disk is far weaker than in a conventional disk. The radiation force is simply the product of the radiation flux and the opacity; where there is no outgoing flux (because there is no internal heat generation), there can be no radiation force. As a result, such 
disks collapse to a state of much greater density (and also column density) than would be predicted by conventional models (Svensson \& Zdziarski 1995: SZ95). They are then supported primarily by gas pressure, and (in the $\alpha$-model) are thermally stable. It is also an immediate corollary that these disks are very nearly isothermal - zero outward flux in an optically thick environment implies constant temperature.

Some analogous work has been done in the past. A number of groups (e.g. Ross \& Fabian 1993; Życki et al. 1994) have computed the spectra of X-ray irradiated disks, but all have assumed the disks are radiation pressure supported. Because disks in which most dissipation is in a corona are much denser than conventional radiation pressure-supported disks, the character of the UV spectrum they produce is quite different from what has been predicted by assuming a conventional disk structure. Others have tried to predict the UV spectra radiated by dense lumps heated by X-rays (Guilbert \& Rees 1988, Ferland \& Rees 1988).

In $\oint 2$. we solve the structure equations for X-ray irradiated disks. The solution of the radiative transfer and vertical structure equations in the X-ray heated skin is described in $\S 3$. and our results are summarized in $\S$. The gravitational stability of the cold disk is discussed in $\S 5$. We conclude in $\S 6$.

\section{The Structure of X-ray Irradiated Accretion Disks}

When there is no internal dissipation (SZ95 treat the case in which the dissipation is shared between the disk and the corona), the main body of the disk is rigorously isothermal, as explained in $\S 1$. The radial structure of geometrically thin isothermal accretion disks is defined by two equations: the conservation of angular momentum

$$
W_{r \phi}=\frac{\dot{M} \omega}{2 \pi} \frac{Q_{N T} C_{N T}^{1 / 2}}{B_{N T} D_{N T}},
$$

and the inter-ring stress prescription, for which we use the $\alpha$-model:

$$
W_{r \phi}=\alpha_{S S} \Sigma_{o} c_{s}^{2} .
$$

Vertical structure is determined by hydrostatic equilibrium:

$$
\frac{\partial P}{\partial z}=-\frac{G M \rho(z)}{R^{2}}\left(\frac{z}{R}\right)\left(\frac{C_{R H}}{B_{R H}}\right)
$$


and the isothermal equation of state. The symbols in these equations represent the mass of the central black hole $(M)$, the mass accretion rate $(\dot{M})$, the Keplerian angular velocity $(\omega)$, the vertically integrated shear stress $\left(W_{r \phi}\right)$, the radial coordinate $(R)$, the vertical coordinate $(z)$, the gas density $(\rho)$, the gas pressure $(P)$, the sound speed in the gas $\left(c_{s}\right)$, the surface mass density

$$
\Sigma_{o}=\int_{0}^{\infty} \rho(z) d z
$$

and the dimensionless stress $\alpha_{S S}$. The general relativistic correction factors are taken from the standard references. The subscript NT refers to the Novikov and Thorne (1973, Page \& Thorne 1974) expressions and the subscript RH refers to the recent corrections presented by Rifferts \& Herrold (1995).

These equations are easily solved. The integrated surface mass density is

$$
\Sigma_{o}=\frac{\dot{M} \omega}{2 \pi \alpha_{S S} c_{s}^{2}} \frac{Q_{N T} C_{N T}^{1 / 2}}{B_{N T} D_{N T}}
$$

and the density profile is Gaussian

$$
\rho(z)=\rho_{o} e^{-\left(z / z_{o}\right)^{2}}
$$

with a scale height of

$$
z_{o}^{2}=\frac{2 k T_{g} R^{3}}{G M m_{p}}\left(\frac{B_{R H}}{C_{R H}}\right)
$$

where $T_{g}$ is the gas temperature in the accretion disk. The normalization of the density,

$$
\rho_{o}=\frac{2}{\sqrt{\pi}} \frac{\Sigma_{o}}{z_{o}}
$$

is set by assuming that all the mass lies in the isothermal inner disk.

It is instructive to express the disk solution in terms of nondimensional parameters

$$
\dot{m}=\frac{\dot{M}}{\dot{M}_{E}} \quad m_{8}=\frac{M}{10^{8} M_{\odot}} \quad r=\frac{R}{R_{s}} \quad T_{5}=\frac{T_{g}}{10^{5} \mathrm{~K}}
$$

where we have introduced the Eddington accretion rate

$$
\dot{M}_{E}=\frac{4 \pi c R_{s}}{\eta \kappa_{e s}}=2.44 \times 10^{26} m_{8} \quad \mathrm{~g} / \mathrm{s}
$$

and the Schwarzschild radius

$$
R_{s}=\frac{2 G M}{c^{2}}=2.95 \times 10^{13} m_{8} \quad \mathrm{~cm} .
$$


The electron scattering opacity is $\kappa_{e s}$. The numerical factors have been calculated by assuming an accretion efficiency of $\eta=0.0572$, which is appropriate for a non-rotating black hole. Using these scalings we find that

$$
\begin{gathered}
\Sigma_{o}=3.38 \times 10^{9} \dot{m} r^{-3 / 2} \alpha_{S S}^{-1} T_{5}^{-1}\left(\frac{Q_{N T} C_{N T}^{1 / 2}}{B_{N T} D_{N T}}\right) \quad \mathrm{gm} / \mathrm{cm}^{2}, \\
z_{o}=5.65 \times 10^{9} T_{5}^{1 / 2} m_{8} r^{3 / 2}\left(\frac{C_{R H}}{B_{R H}}\right)^{-1 / 2} \mathrm{~cm},
\end{gathered}
$$

and

$$
\rho_{o}=0.68 \dot{m} m_{8}^{-1} r^{-3} \alpha_{S S}^{-1} T_{5}^{-3 / 2}\left(\frac{Q_{N T} C_{N T}^{1 / 2}}{B_{N T} D_{N T}}\right)\left(\frac{C_{R H}}{B_{R H}}\right)^{1 / 2} \mathrm{gm} / \mathrm{cm}^{3} .
$$

There are two important things to note about these results. First, the total surface mass density is many orders of magnitude larger than the penetration depth of the hard X-rays. Consequently, supposing the bulk of the disk to be isothermal and gas pressure-supported is an excellent approximation. Second, the gas density of the isothermal disk is very much larger than the $\sim 10^{-10} \mathrm{gm} \mathrm{cm}^{-3}$ typical of conventional radiation pressure supported disks (e.g., SS73).

\section{The X-ray Heated Skin}

Few X-rays can penetrate much greater than the Compton depth $\Sigma_{C} \simeq 0.5 \mathrm{gm} \mathrm{cm}^{-2}$. On the other hand, as shown by equation 12, these disks are extremely optically thick to Compton scattering. Consequently, there are essentially no X-rays below $z / z_{o} \simeq\left[\ln \left(\Sigma_{o} / \Sigma_{C}\right)\right]^{1 / 2} \simeq 3-4$. The exact number of scale heights above the midplane where the X-ray-heated skin begins depends only slightly on parameters. In this estimate, and all the numerical work, we set $\alpha_{S S}=0.1$ for definiteness, but it hardly affects the results at all.

\subsection{Differential Equations and Constraints}

Inside the X-ray-heated skin, the temperature, pressure, and radiation intensity are determined as functions of altitude by solving the differential equations for radiative transfer,

$$
\frac{\partial}{\partial \Sigma}\left\{\frac{1}{\kappa_{T, \nu}} \frac{\partial\left(f_{\nu} J_{\nu}\right)}{\partial \Sigma}\right\}=\kappa_{a, \nu} J_{\nu}-\frac{1}{4 \pi} \varepsilon_{\nu}
$$


hydrostatic equilibrium

$$
\frac{\partial P}{\partial \Sigma}=g(\Sigma)+\frac{H(\Sigma)}{3 c}-\frac{4 \pi}{c} \int d \nu \frac{\partial\left(f_{\nu} J_{\nu}\right)}{\partial \Sigma}
$$

and the surface mass density

$$
\frac{\partial z}{\partial \Sigma}=-\frac{1}{\rho}
$$

subject to the local constraints of radiative equilibrium

$$
\int d \nu\left\{4 \pi \kappa_{a, \nu} J_{\nu}-\varepsilon_{\nu}\right\}+H(\Sigma)=0
$$

and charge conservation

$$
n_{e}=\sum_{k} \sum_{l=1}^{L_{k}} l n_{l k}
$$

Equations 15, 16, 17, 18, 19 are solved for the mean ultraviolet intensity $\left(J_{\nu}\right)$, gas pressure $(P)$, gas temperature $(T)$, electron density $\left(n_{e}\right)$ and vertical coordinate $(z)$ as functions of the surface mass density. The other symbols represent the X-ray heating rate $(H(\Sigma)$, see \$3.2. , total opacity $\left(\kappa_{T, \nu}\right)$, the absorptive opacity $\left(\kappa_{a, \nu}\right)$, the emissivity $\left(\varepsilon_{\nu}\right)$, the variable Eddington factors $\left(f_{\nu}\right)$, the number density of ion species $k$ in ionization state $l\left(n_{l k}\right)$ and the maximum ionization state of species $k\left(L_{k}\right)$.

We assume that the disk is geometrically thin and neglect self-gravity of the disk. Under these assumptions, the gravitational acceleration of the material in the disk is (Rifferts \& Herrold 1995)

$$
g(\Sigma)=\frac{G M z(\Sigma)}{R^{3}}\left(\frac{C_{R H}}{B_{R H}}\right) .
$$

The gravitational force of the underlying isothermal disk can contribute to the net acceleration of material in the skin but the contribution is small (see the discussion in \$2.). The momentum of the absorbed X-rays is transferred to the gas, resulting in an additional force directed towards the disk midplane. The gradient of the hard X-ray radiation pressure is

$$
\frac{\partial P_{r, x}}{\partial \Sigma}=-\frac{H(\Sigma)}{3 c}
$$

in the Eddington approximation, which we have used to solve for $H(\Sigma)$ (§3.2.).

The total opacity includes bremsstrahlung, photoionization and electron scattering. We neglect all line opacities. The expression for the free-free opacity is taken from Rybicki \& Lightman (1979) and the general expression for the photo-ionization opacity is from Mihalas (1978). The bound-free oscillator strengths of HI and HeII are calculated using the analytic formulae for hydrogen-like atoms (e.g., Mihalas 1978) and the HeI oscillator 
strengths are from Bell \& Kingston (1967). We calculate the number densities of electrons, HI, HII, HeI, HeII and HeIII assuming Saha equilibrium, i.e., local thermodynamic equilibrium, and hydrogen and helium abundances of $\mathrm{X}=0.9$ and $\mathrm{Y}=0.1$, respectively. Explicit photoionization and recombination are not included in the calculation of the ionization state.

We compute the emissivity using the corresponding continuum cooling processes: bremsstrahlung and HI, HeI, and HeII radiative recombination. The gas density in the X-ray heated layer is high enough that we expect resonance line cooling to be suppressed. Resonance lines are thermalized when the collisional deexcitation rate is greater than the product of the radiation rate and the photon escape probability. Neglecting stimulated emission, this occurs when the fractional abundance of the lower state $X_{l}$ satisfies the inequality

$$
X_{l}>10^{-9} n_{e 18}^{-1} N_{H 22}\left(\frac{m_{A}}{m_{p}}\right)^{-1 / 2}\left(h \nu / I_{H}\right)^{3},
$$

where $n_{e 18}$ is the electron density in units of $10^{18} \mathrm{~cm}^{-3}, N_{H 22}$ is the column density of $\mathrm{H}$ atoms in units of $10^{22} \mathrm{~cm}^{-2}$, and $m_{A, p}$ the masses of the atom and proton, respectively. These scalings were chosen because the gas density at 3.5 scale heights from the midplane is typically $n_{e} \sim 10^{18} \mathrm{~cm}^{-3}$ and $2 \mathrm{keV}$ photons are absorbed at a column density $N \sim 10^{22}$ $\mathrm{cm}^{-2}$. When the temperature is $\sim 10^{5} \mathrm{~K} \mathrm{UV}$ and EUV lines are the important potential coolants, but the only lines which contribute to the cooling are those with very low abundance lower states.

By contrast, the density in the atmosphere of an irradiated radiation pressure supported disk is characteristically four orders of magnitude lower. This means that the ionization parameter is so much greater that a thermal runaway is triggered, and soft X-ray lines are the most important transitions for cooling. Due to the combination of lower density and higher radiation rates, emission lines are much more important in the thermal balance in this context than in our limit of the zero internal dissipation disk.

In principle, the complete angular dependent radiative transport problem can be solved by introducing variable Eddington factors (e.g., Mihalas 1978). The computational overhead associated with an iterative calculation of the Eddington factors is fairly large and, given the other approximations we have made, probably unwarranted. Instead, we adopt a simple parametrization

$$
f_{\nu}=\frac{1+\tau_{\nu}^{2}}{1+3 \tau_{\nu}^{2}}
$$

where

$$
\tau_{\nu}=\int \kappa_{T, \nu} d \Sigma
$$


which has the correct limiting behavior (G. S. Miller, private communication). We perform an iterative calculation of $f_{\nu}$ for one set of disk parameters in $\$ 4.2 .2$. and use this solution to test the accuracy of the analytic prescription.

The complete system of equations is solved by transforming the differential equations into finite-difference equations, linearizing with respect to all variables, and relaxing from

an initial solution. We solve the structure equations for a grey atmosphere with a shooting procedure (Press, et al. 1992) and use this solution as a first guess for the relaxation method. The only significant difference between our work and the standard stellar atmosphere calculation (e.g., Mihalas 1978, Rybicki 1971) is the non-zero heating rate (see eq. 18).

\subsection{The X-ray Heating Rate}

We assume that all the energy dissipated due to accretion at radius $R$ is released as heat in the corona. The rate per unit area is

$$
Q_{d i s}=\frac{3 G M \dot{M}}{8 \pi R^{3}}\left(\frac{Q_{N T}}{B_{N T} C_{N T}^{1 / 2}}\right)
$$

and this is converted into an X-ray flux by inverse Compton scattering of the UV photons emerging from the disk (HM93). One-half of this flux illuminates the underlying disk. In terms of the dimensionless parameters defined in eq. 9

$$
Q_{d i s}=1.50 \times 10^{19} \dot{m} m_{8}^{-1} r^{-3}\left(\frac{Q_{N T}}{B_{N T} C_{N T}^{1 / 2}}\right) \operatorname{ergs~cm}^{-2} \mathrm{~s}^{-1} .
$$

The spectrum of the illuminating flux is taken to be

$$
S(E)=S_{o} E^{-\alpha} \exp -E / E_{c}
$$

where $E_{c}=10 \mathrm{keV}$. A self-consistent calculation of the X-ray spectrum is beyond the scope of this paper, so we simply assume $\alpha=0.9$, as is commonly observed (Zdziarski et al. 1995). The exponential cut-off is meant to mimic the sharp rise in the X-ray albedo above $10 \mathrm{keV}$ (Lightman \& White 1988). As a result of the high albedo for harder photons, they are mostly reflected and do not contribute to the heating rate. Still harder photons $(E>100 \mathrm{keV})$ lose much of their energy in recoil, but because there are many uncertainties in our knowledge of the high energy spectrum in AGN (Zdziarski et al. 1995), and because the expected contribution to the net heating is small and depends sensitively on the high 
energy spectrum (P. Zýcki, private communication), we neglect this contribution. We also suppose that there is no sub-structure to the corona; the local heating rate could vary substantially if the dissipation is concentrated in active regions (e.g., Haardt, Maraschi \& Ghisellini 1994). These complications will be addressed in later work.

Given this simple parametrization, the heating rate is a well-defined function of the surface mass density

$$
H(\Sigma)=\int \kappa_{x, e f f}(E) S(E) e^{-\kappa_{x, e f f}(E) \Sigma} d E
$$

where the effective X-ray absorption opacity is defined as (Rybicki \& Lightman 1979)

$$
\kappa_{x, e f f}(E)=\left[3 \kappa_{x}(E)\left\{\kappa_{x}(E)+\kappa_{e s}\right\}\right]^{1 / 2} .
$$

and $\kappa_{e s}$ is the electron scattering opacity.

The X-ray opacity in this energy range is dominated by photoionization of oxygen and iron (e.g., London, McCray \& Auer 1981). The threshold energy for K-shell photoionization depends upon the ionization state of the gas (Daltabuit \& Cox 1972) which is largely determined by the incident X-ray flux (Lightman \& White 1988, Zýcki, et al. 1994). A careful calculation of the ionization balance of the heavy elements is beyond the scope of this paper. Instead, we assume threshold energies of $0.8 \mathrm{keV}$ and $8.0 \mathrm{keV}$ for oxygen and iron, respectively, and solar abundances of these elements. The X-ray opacity can then be estimated as

$$
\kappa_{x}(E)= \begin{cases}47.9\left(\frac{0.8 \mathrm{keV}}{E}\right)^{3} \mathrm{~cm}^{2} \mathrm{~g}^{-1} & 0.8<E<8.0 \mathrm{keV} \\ 0.17\left(\frac{8.0 \mathrm{keV}}{E}\right)^{3} \mathrm{~cm}^{2} \mathrm{~g}^{-1} & E>8.0 \mathrm{keV}\end{cases}
$$

using absorption cross-sections for hydrogen-like atoms (Rybicki \& Lightman 1979). This approximate description slightly underestimates the absorptive opacity in most of the $\mathrm{X}$-ray-heated atmosphere, where most medium-Z atoms retain some valence shell electrons.

The expression for $H(\Sigma)$ (eq. 28) is equivalent to a two-stream solution for the radiative transfer equation in the Eddington approximation. This approximation is reasonable because the incident hard X-rays are nearly isotropic in the lower hemisphere and electron scattering in the disk will tend to isotropize the X-rays. The heating rate at lower $\Sigma$ is probably underestimated in this approximation because X-rays incident at large angles will be absorbed at smaller $\Sigma$.

The local heating rate is calculated by integrating equation 28 numerically and the results for the assumed X-ray spectrum are plotted in fig 11. The overall scale of the heating rate depends upon the accretion parameters, but the $\Sigma$ dependence is the same for all the models. At low column densities, the heating rate is almost independent of depth because 
all X-ray frequencies are optically thin. When $0.01 \mathrm{gm} \mathrm{cm}^{-2} \lesssim \Sigma \lesssim 1.0 \mathrm{gm} \mathrm{cm}^{-2}$,

$$
H(\Sigma) \propto E_{*} \kappa_{x, \text { eff }}\left(E_{*}\right) S\left(E_{*}\right) \propto \Sigma^{-(2+\alpha) / 3} \simeq \Sigma^{-1}
$$

because photons of energy $E_{*}$ are absorbed at the depth $\Sigma\left(E_{*}\right) \propto E_{*}^{3}$. At $\Sigma \gg 1.0 \mathrm{gm} \mathrm{cm}^{-2}$, the $\mathrm{X}$-ray flux is exponentially attenuated and the heating rate falls rapidly. The analytic approximation

$$
H(\Sigma) \simeq 3.3 Q_{\text {dis }}\{1-\exp (-0.025 / \Sigma)\} \exp (-\Sigma / 11.0)
$$

is also plotted in fig. 1. Here $\Sigma$ is measured in units of $\mathrm{gm}^{-2}$.

\subsection{Boundary Conditions}

The boundary between the X-ray heated skin and the isothermal disk is set at $\Sigma_{1}=50.0 \mathrm{gm} \mathrm{cm}^{-2}$. The X-ray heating rate at the boundary is negligible $\left(\Sigma_{1} \gg 1 \mathrm{gm} \mathrm{cm}^{-2}\right)$ and we assume no dissipation in the cold disk, so

$$
F_{\nu}=\frac{4 \pi}{\kappa_{T, \nu}} \frac{\partial\left(f_{\nu} J_{\nu}\right)}{\partial \Sigma}=0
$$

at the inner boundary. The gas temperature is taken to be continuous across the boundary and the inner boundary conditions on the gas pressure and vertical coordinate are fixed by requiring that the numerical solution for the skin match the analytic solution for the isothermal disk at $\Sigma_{1}$.

The X-ray heated skin has a sharp outer boundary because a thermal runaway occurs at that point (see $\S 4.1$ for a discussion of its physical origin). We designate the column density at this runaway $\Sigma_{2}$. Its location is found by bisection. We guess $\Sigma_{2}$ and attempt a solution. If the code converges to a thermally stable solution, $\Sigma_{2}$ is decreased; if the code fails to converge, it is increased. This is repeated until the fractional change in $\Sigma_{2}$ is less than a few percent. We tested a number of methods for determining the location of the thermal runaway and found that the most sensitive test was the convergence of the code.

The outer boundary condition on the mean UV intensity is usually set by assuming that there is no incident intensity at the boundary (Mihalas 1978). However, in our situation this is not a valid approximation because $\Sigma_{2}$ (or the Compton depth of the corona) may be large enough to reflect a significant amount of radiation back towards the skin. Instead, we assume that the UV flux remains constant at $\Sigma<\Sigma_{2}$ and that $\kappa_{e s} \gg \kappa_{a, \nu}$. We neglect any contribution from the hot corona to the Thomson depth at $\Sigma<\Sigma_{2}$. We also neglect 
(except in the global sense that this is the origin of the X-rays) any frequency change in the UV photons caused by scattering off gas at $\Sigma<\Sigma_{2}$. Under these assumptions, there is an analytic relation between the mean intensity and the flux (Mihalas 1978) which provides our boundary condition

$$
h_{\nu} J_{\nu}=\frac{1}{\kappa_{T, \nu}} \frac{\partial\left(f_{\nu} J_{\nu}\right)}{\partial \Sigma}
$$

where

$$
\begin{gathered}
h_{\nu}=\frac{1}{3} \cdot \frac{1}{\tau_{2, \nu}+2 / 3-E_{2}\left(\tau_{2, \nu}\right) / 3+E_{3}\left(\tau_{2, \nu}\right) / 2}, \\
\tau_{2, \nu}=\kappa_{T, \nu} \Sigma_{2}
\end{gathered}
$$

and $E_{i}$ are exponential integrals. In practice, the final value of $\tau_{2, \nu} \ll 1$ and eq. 35 reduces to the free streaming value, $h_{\nu} \simeq 1 / \sqrt{3}$. However, $\tau_{2, \nu} \gtrsim 1$ for many of the intermediate steps.

\section{Results}

\subsection{The Vertical Structure of the X-ray Heated Skin}

The inner edge of the X-ray heated skin is 3-4 scale heights from the midplane of the isothermal disk. The thickness of the skin increases slightly with radius but remains $\lesssim 10 \%$ of the height of the inner edge (fig. 2a). The thin disk approximation is valid at all radii and the geometry of the disk can be determined from the isothermal disk solution (§2.). The density profile in the isothermal disk is Gaussian so the gas pressure at the inner edge of the skin is much smaller (typically by $\sim$ four orders of magnitude) than the central disk pressure. This pressure is still much larger than the pressure in a fully radiation pressure-supported disk, however. Throughout most of the skin, the radiation force of the UV photons is great enough to balance gravity and the force of the incident X-ray flux. The gas pressure is therefore nearly constant for $\Sigma \lesssim 10 \mathrm{gm} \mathrm{cm}^{-2}$ (see fig. 2 $\mathrm{b}$ ). Both the $\mathrm{X}$-ray and UV fluxes drop to zero at $\Sigma \gtrsim 10 \mathrm{gm} \mathrm{cm}^{-2}$ and a gas pressure gradient forms to support the disk against gravity. The gas pressure profile joins smoothly to the isothermal disk solution at $\Sigma_{1}$.

The radiative cooling rate is equal to the $\mathrm{X}$-ray heating rate when the gas is in radiative equilibrium. Therefore, the gas temperature must be greater than the UV radiation temperature because the cooling rate is $\propto T_{g}^{4}-T_{r}^{4}$, where

$$
T_{r}=\left(\frac{\pi J}{\sigma}\right)^{1 / 4}
$$


and $J$ is the frequency integrated mean intensity of the UV radiation field. The actual gas temperature profile is determined by three competing effects. First, the mean intensity, and $T_{r}$, decrease from the bottom of the skin to the surface because of the net outward flux. Second, the X-ray heating rate increases from zero at the bottom of the skin to its maximum value at the top. Therefore, $T_{r}=T_{g}$ at $\Sigma_{1}$ and the difference between the two temperatures increases steadily until $T_{g} \gg T_{r}$ at the upper edge of the skin. Third, the gas temperature gradient changes when an opacity jump, such as the Lyman edge, becomes transparent (Mihalas 1978, p. 206). The gradient can even change sign in some cases, but not in all cases (see fig. Q). The relative importance of these three effects depends upon the local gas pressure and temperature. We find that $T_{g}$ generally increases from the midplane outward but the increase is not always monotonic.

An example of a typical temperature profile is presented in fig. 2 2 c. The gas is optically thick at all frequencies when $\Sigma \gtrsim 10 \mathrm{gm} \mathrm{cm}^{-2}$ and the temperature gradient

$$
\frac{\partial T}{\partial \Sigma} \sim 0 .
$$

The gradient is slightly greater than 0 (i.e., $T$ increases inward) at $\Sigma \gtrsim 15 \mathrm{gm} \mathrm{cm}^{-2}$, and slightly less than 0 (i.e., $T$ increases outward) for $10 \mathrm{gm} \mathrm{cm}^{-2} \lesssim \Sigma \lesssim 15 \mathrm{gm} \mathrm{cm}^{-2}$. The $\mathrm{X}$-ray heating rate increases rapidly at $\Sigma \lesssim 10 \mathrm{gm} \mathrm{cm}^{-2}$ and $T_{g}$ rises to maintain thermal equilibrium. In this example, the photosphere for frequencies below the Lyman edge is at $\Sigma \simeq 0.4 \mathrm{gm} \mathrm{cm}^{-2}$, so the temperature gradient changes sign at this point. The sign flips back at the photosphere for frequencies just above the Lyman edge. The gradient remains positive at smaller $\Sigma$ and the thermal runaway starts at $\Sigma=0.012 \mathrm{gm} \mathrm{cm}^{-2}$.

The upper boundary of the UV reprocessing region is set by the thermal runaway. The temperature runs away, causing the code to fail to converge, when the X-ray ionization parameter

$$
\Xi=\frac{F_{X}(\Sigma)}{P c} \gtrsim 1,
$$

where $F_{X}(\Sigma)$ is the X-ray flux at $\Sigma$. When $\Xi$ exceeds this critical value, bremsstrahlung and recombination cannot cool the gas, and its temperature rises toward the Compton temperature (Krolik, McKee \& Tarter 1981; Voit \& Shull 1988; Ferland \& Rees 1988). The exact mass column of the thermal runaway $\left(\Sigma_{2}\right)$ depends upon $\dot{m}, m_{8}$ and $r$, but in general $0.01 \mathrm{gm} \mathrm{cm}^{-2} \lesssim \Sigma_{2} \lesssim 1 \mathrm{gm} \mathrm{cm}^{-2} . \Sigma_{2}$ is never smaller than $\simeq 0.01 \mathrm{gm} \mathrm{cm}^{-2}$ because the skin is optically thin to X-rays above that point and the gas pressure doesn't change much over such a small column density. On the other hand, $\Sigma_{2}$ is always less than about one Compton depth because the X-rays are exponentially attenuated beyond that point.

Typically $\Sigma_{2}$ rises sharply at larger radii. A good example of this effect is seen in the model $m_{8}=2.7$ and $\dot{m}=0.3$ (see fig. $⿴$ ). The reason for this sharp rise is that HeII 
recombination provides much of the cooling at columns $0.1 \lesssim \Sigma \lesssim 1$ at smaller radii, but the lower temperatures at larger radii mean that little HeIII is formed there.

Within the framework of the model it is possible to estimate $f_{U V}$, the fraction of the incident X-ray flux which is reradiated in the UV. This quantity is determined by how much $\mathrm{X}$-ray flux is either absorbed or reflected by the material in the thermal runaway layer at column densities smaller than $\Sigma_{2}$. We estimate $f_{U V}$ by the somewhat crude prescription of assuming that the opacity of the warm layer is unchanged by the thermal runaway. A more careful treatment would allow for the reduction in photoelectric opacity due to the higher ionization states found there. Nonetheless, we believe our global estimate of $f_{U V}$ is not too bad because the shielding effect is only of major importance when $\Sigma_{2}$ is at least $\sim 1 \mathrm{gm} \mathrm{cm}^{-2}$. When $\Sigma_{2}$ is that large, it hardly matters whether the disk proper is shadowed by photoelectric opacity or Compton scattering.

The general trend of $f_{U V}$ is to decrease with increasing $\dot{m}$ because $\Sigma_{2}$ increases. The gas pressure in the skin is

$$
P_{s} \sim P_{o}\left(\frac{1 \mathrm{gm} \mathrm{cm}^{-2}}{\Sigma_{o}}\right) \propto m_{8}^{-1} .
$$

On the other hand, $F_{X} \propto Q_{d i s} \propto \dot{m} / m_{8}$ at fixed $r$. Consequently, $\Xi \propto \dot{m}$ at the upper boundary of the skin. Thus, as $\dot{m}$ increases, the X-ray flux is able to trigger a thermal runaway at larger column densities and over a broader range of radii. When $\dot{m} \lesssim 0.003$ the $\mathrm{X}$-ray flux is never large enough to cause a runaway (see fig. (4).

In table 11 we list

$$
f_{U V}=\frac{\int r d r F_{X}\left(r, \Sigma_{2}\right)}{\int r d r F_{X}(r, 0)},
$$

where $F_{X}(r, \Sigma)$ is the X-ray flux at a mass column $\Sigma$ in the ring at radius $r$. We find that $f_{U V}$ is 1.0 at $\dot{m}=0.003$ and decreases to 0.62 at $\dot{m}=0.3$. The column density of the runaway is insensitive to $m_{8}$ and $f_{U V}$ varies only slightly when $m_{8}$ changes by three orders of magnitude. We caution that these numerical values also depend on the specific X-ray spectral shape we have chosen.

The material above $\Sigma_{2}$ might influence the emergent spectrum by radiating soft X-rays and by Comptonizing the UV flux leaving the disk atmosphere. We can estimate the gas temperature there as

$$
T_{s x} \simeq \frac{J_{u v} T_{r}+J_{x} T_{x}}{J_{u v}+J_{x}}
$$

(Begelman, McKee \& Shields 1988), where $J_{u v, x}$ are the mean intensities of the UV and incident X-rays, respectively, and $T_{r, x}$ are the corresponding Compton temperatures. Although we discount the effect of hard X-rays for heating the disk, they are effective for 
Compton heating, at least up to $\simeq 100 \mathrm{keV}$, beyond which the Klein-Nishina reduction in the scattering rate curtails their contribution. If the spectrum is $\propto E^{-0.9}$ over many orders of magnitude in photon energy, $T_{x} \simeq 3 \times 10^{7} \mathrm{~K}$. As we have already estimated, $T_{r} \lesssim 10^{5} \mathrm{~K}$. The UV and X-ray intensities are comparable in the skin, so the additional cooling provided by scattering of the UV photons will reduce the gas temperature to $T_{s x} \simeq T_{x} / 2 \simeq 1.5 \times 10^{7} \mathrm{~K}$, roughly independent of radius out to the point where the heating is insufficient to maintain a corona at high temperature.

The emission measure $(E M)$ of the soft X-ray corona is dominated by the regions at larger radius where $\Sigma_{2} \sim 1 \mathrm{gm} \mathrm{cm}^{-2}$. We estimate

$$
E M \sim 2 \times 10^{65} m_{8} r^{1 / 2} T_{5}^{1 / 2}\left(\frac{\Sigma_{2}}{1 \mathrm{gm} \mathrm{cm}^{-2}}\right)^{2}\left(\frac{\Delta z}{z_{o}}\right)^{-1} \mathrm{~cm}^{-3}
$$

where $\Delta z$ is the thickness of the corona. We expect $\Delta z \lesssim 0.1 z_{o}$ and so the associated bremsstrahlung luminosity $\left(\sim 10^{43} m_{8} \mathrm{erg} \mathrm{s}^{-1}\right)$ is much smaller than the total disk luminosity unless $\dot{m} \lesssim 10^{-3}$. Similarly, the importance of Comptonization to the spectrum depends on the Compton $y$-parameter, $\left(\tau_{T}+\tau_{T}^{2}\right) k T_{s x} /\left(m_{e} c^{2}\right)$, where $\tau_{T}$ is the Thomson depth of the layer. Because $\tau_{T}$ is at most $\sim 1$ and we expect $k T_{s x} /\left(m_{e} c^{2}\right) \sim 10^{-2}$, this, too, is unlikely to alter the emergent spectrum. We elaborate on the effects of Comptonization on the UV spectrum in $\$ 4.2 .3$.

\subsection{The Reprocessed Ultraviolet Spectrum}

\subsubsection{The Face-On Disk}

We calculated integrated spectra for face-on disks over a range of $\dot{m}$ and $m_{8}$ chosen to facilitate comparisons with previous calculations of disk spectra, namely luminosities ranging from 0.003 to 0.3 of the Eddington luminosity and central masses of $2.7 \times 10^{7} M_{\odot}$ to $2.7 \times 10^{9} M_{\odot}$. In each case, we divided the disk into annuli spaced at (roughly) equal intervals in $\Delta r / r$ and the local structure and spectrum were calculated. The radii ranged from the inner radius $r_{i}=3.5$ to some outer radius at which the gas temperature was so low that the contribution from the outermost radius to the flux near the Lyman edge became negligible. This occurs at $r \gtrsim 20$ where $T_{\text {eff }} \lesssim 1.5 \times 10^{4} \mathrm{~K}$ (eq. A5). Finally, the local spectra are added using the method described by LN89, which accounts for limb-darkening, Doppler boosting, and relativistic aberration of the emergent flux. We neglect the general 
relativistic bending of light rays in the gravitational field of the central black hole (e.g., Cunningham 1975). A variety of face-on spectra are plotted in (figs. 78, 8). Note that we define $L_{\nu}(\theta)$ as $2 \pi \times$ the luminosity per solid angle at an angle $\theta$ from the disk axis.

In the simplest treatment of predicted accretion disk spectra, the dynamics are approximated as Newtonian, and the spectra of individual rings are assumed to be blackbodies. These approximations lead to a temperature profile $\propto r^{-3 / 4}$ and an integrated spectrum whose shape is roughly $L_{\nu} \propto \nu^{1 / 3} \exp \left(-h \nu / k T_{*}\right)$, where $T_{*}$ is the temperature of the innermost ring. It is clear from these figures that such an approximation is not a very good description of our predicted spectra. At low frequencies, there is considerable spectral curvature, and strong Lyman edge features substantially modify the shape of the exponential cut-off at high frequencies.

Three different effects alter the spectral shape at frequencies below the Lyman edge. First, relativistic effects flatten the temperature profile in the inner rings (Novikov \& Thorne 1973). This softens the spectrum relative to the Newtonian approximation. Second, the presence of a large emission feature at the Lyman edge implies that a smaller fraction of the reprocessed flux emerges at frequencies below the Lyman edge. This depresses the general level of the near-UV continuum. Third, at frequencies below the Lyman edge, the mass column of the UV photosphere increases with increasing frequency. The flux at lower frequencies then comes from hotter gas because the gas temperature typically increases as $\Sigma$ decreases. This softens the continuum spectrum relative to the black body approximation.

The low-frequency spectrum becomes harder with increasing hard X-ray flux, i.e., $\dot{m} / m_{8}$. The disk temperature is roughly proportional to the effective temperature of the $\mathrm{X}$-ray flux, so the peak of the disk spectrum shifts from $10^{15} \mathrm{~Hz}$ in the coldest disks to $\gtrsim 10^{15.5} \mathrm{~Hz}$ in the hottest. To quantify this effect, we define $\alpha_{15}=d \log L_{\nu} / d \log \nu$ at $\log \nu=15.0$. The value of this quantity for each model we computed is displayed in table 1 . The smallest $\alpha_{15}$ we found was $-1.6\left(\dot{m} m_{8}^{-1} \sim 10^{-3}\right)$; the largest was $0.8\left(\dot{m} m_{8}^{-1} \sim 10^{-1}\right)$.

As the previous paragraphs explain, some, but not all, these effects are due to the external irradiation. In particular, the temperature gradient effect acts the opposite way when the dissipation is inside the disk rather than outside. An improved calculation of the spectrum of a conventional disk with internal dissipation methods yields a predicted $\alpha_{15} \simeq 1$ (Sincell \& Krolik 1996).

We found a strong Lyman edge emission emission feature over the entire parameter range we studied (see figs. 6, 7 and 8). This, too, is due to the temperature increasing upward (22d, 20). The larger opacity above the edge than below causes the Lyman continuum photosphere to lie higher in the atmosphere than the photosphere at frequencies just below 
the edge. The higher temperature at the Lyman continuum photosphere naturally leads to a greater emergent flux, i.e. an emission feature (fig. 6). Additional emission features appear at the HeI and HeII edges in some of the integrated spectra. The smearing of the edges is caused by Doppler boosting of radiation emitted at small $r$.

The amplitude of the Lyman edge feature increases with the central mass (fig. (7) because $P_{s} \propto m_{8}^{-1}$ (eq. 40). Low pressure gas cools less efficiently because both the bremsstrahlung and photoionization opacities are proportional to $P_{s}$. Inefficient cooling leads to larger temperature inversions in the skin. The ratio $\Sigma_{L y+} / \Sigma_{L y-}$ remains roughly constant because the ratio of the opacities is independent of $P_{s}$. Therefore, $T\left(\Sigma_{L y+}\right) / T\left(\Sigma_{L y-}\right)$ increases with $m_{8}$ and the amplitude of the Lyman edge feature increases. The amplitude of the edge feature is nearly independent of $\dot{m}$ (fig. 8).

\subsubsection{Angular Distribution of the Emergent Flux}

We incorporated an iterative calculation of the variable Eddington factors (Auer \& Mihalas 1970) into the $\dot{m}=0.03, m_{8}=2.7$ model. The solution for the Eddington factors $\left(f_{\nu}\right)$ and the mean intensity is equivalent to the exact solution for the angular distribution of the spectral intensity. The angular distribution of the emergent flux can be determined by a formal solution of the transfer equation with the known source function. The surface Eddington factors (eq. 35) cannot be computed iteratively because we do not have a solution at $\Sigma<\Sigma_{2}$.

The accuracy of the analytic approximation for the Eddington factors (eq. 23) was tested by comparison with the exact solution. The radiation field is isotropic when $\tau \gg 1$ and $f_{\nu}=0.33$, in good agreement with the analytic approximation. However, when $\tau \ll 1$ we find that $f_{\nu} \lesssim 0.5$. The approximate $f_{\nu}$ assumes that the radiation is collimated perpendicular to the accretion disk so that $f_{\nu}=1$. Fortunately, the errors introduced by our assumed $f_{\nu}$ are small. The approximate solution underestimates the true gas temperature by $\lesssim 5 \%$ at $\Sigma \lesssim 1 \mathrm{gm} \mathrm{cm}^{-2}$ and overestimates the temperature by $\lesssim 10 \%$ at larger $\Sigma$. The gas pressure is underestimated by $\lesssim 10 \%$ at all $\Sigma$. The differences in the UV spectrum depend on frequency and never exceed 10\%. The approximate solution overestimates the mean intensity below the Lyman edge and above the HeII edge and underestimates $J_{\nu}$ between these frequencies. As a consequence, the amplitude of the Lyman edge feature is larger in the exact solution.

The observed flux from an accretion disk around a massive black hole depends upon

the disk inclination angle. First, as discussed in the previous paragraph, the emergent flux 
has an intrinsic angular distribution in the local rest frame (see below). Second, there are a number of relativistic effects acting upon photons emitted from material orbiting near the black hole. Relativistic orbital motion boosts their frequencies and collimates their directions, while the strong gravitational field bends their trajectories and imposes a gravitational redshift on their frequencies as viewed at large distance (Cunningham 1975). The boosting and beaming due to orbital motion tend to be stronger than the gravitational effects, so the net result is to strengthen the radiation emitted close to the equatorial plane from the innermost rings of the disk (Cunningham 1975, Laor, Netzer \& Piran 1990). These effects are stronger in rotating black holes than in non-rotating black holes because the disk extends closer to the event horizon in the former case (Laor, et al. 1990). For this reason, the impact of general relativistic effects is small in our (non-rotating black hole) models.

We calculated the integrated spectrum of the $\dot{m}=0.03, m_{8}=2.7$ disk for three disk inclination angles. For each inclination, the disk is divided into several annuli ( $\$ 4.2 .1$.) and each annulus subdivided into several sectors. The radiative transfer equation is solved for the spectral intensity at the appropriate rest-frame inclination angle using the exact source function and the results are summed over all annuli and sectors. As before, Doppler boosting, relativistic aberration and the gravitational redshift are included, but we neglect the general relativistic bending of the light rays by the central black hole.

At low frequencies, the emergent intensity is limb-darkened (fig. 9). Due to the low opacity below the Lyman edge, the photosphere for these frequencies tends to lie relatively deep inside the atmosphere, where the temperature gradient is small or positive (i.e. temperature decreases upward). Consequently, the angular dependence is determined largely by the usual limb-darkening influence of scattering. However, at frequencies well above the Lyman edge, the intensity is more nearly isotropic. Several effects combine to explain this change. Because of the greater opacity, the photosphere in this range lies higher in the atmosphere, where the temperature tends to increase outward. Therefore, photons traveling well away from the axis can only depart from relatively high altitude, where the temperature is comparatively high. In addition, because these frequencies are radiated almost exclusively by the innermost rings of the disk, relativistic boosting and beaming are especially important. The decreasing degree of limb-darkening with increasing frequency causes the feature at the Lyman edge to strengthen with disk inclination.

We expect the relative limb-brightening of the ionizing continuum to increase with central mass and, if $L / L_{E}$ is approximately constant, disk luminosity. This is because temperature gradients increase with $m_{8}$ (§世.2.1.).

Doppler broadening of the Lyman edge increases with disk inclination. The typical line of sight to an edge-on disk is nearly parallel to the disk rotation velocity so the Doppler 
boost is maximized. The Lyman edge is formed at $r \sim 10-20$, where $\beta_{\phi} \sim 0.2$, so the edge is spread over a range $\frac{\Delta \nu}{\nu} \lesssim 0.2$ (fig. 9). The peak of the emergent flux shifts upward by the same amount. An edge-on disk will not appear to have an emission feature at the Lyman edge, but the continuum slope becomes harder going from frequencies below the Lyman edge to higher frequencies. This result is consistent with the conclusions of Laor (1992).

\subsubsection{Comptonization of the UV Radiation}

The reprocessed UV radiation is inverse Compton scattered by the relativistic electrons in the coronae. We calculate the Comptonized UV spectrum for scattering by a relativistic Maxwell-Boltzmann distribution of electrons

$$
\left.N(\gamma)=\frac{1}{\Theta K_{2}(1 / \Theta)} \gamma\left(\gamma^{2}-1\right)\right)^{1 / 2} \exp (-\gamma / \Theta)
$$

where $\Theta=k T_{c} / m_{e} c^{2}$, the coronal temperature is $T_{c}$ and $K_{2}$ is the second order modified Bessel function. This distribution is normalized so that

$$
\int d \gamma N(\gamma)=1
$$

and we assume a Thomson depth, $\tau_{c}$, for the corona. We further assume that each photon has a probability $\tau_{c}<1$ of being scattered in the corona and that each photon is scattered at most one time. The spectrum of the scattered radiation is calculated using the formulae for isotropic electron and photon distributions (Rybicki \& Lightman 1979). The small corrections due to anisotropy of the incident UV radiation field were neglected (Haardt 1993). Higher order scatterings determine the shape of the high energy

spectrum (Pozdnyakov, Sobol \& Sunyaev 1976) so we plot the results over a limited range of frequencies near the Lyman edge (figs. 10 and 11).

The spectrum of the Comptonized UV flux is determined primarily by the Thomson depth of the corona, $\tau_{c}$. Inverse Compton scattering in an optically thin corona has a negligible effect on the spectrum of the UV continuum because few photons are scattered (fig. 10). Comptonization in an optically thick corona $\left(\tau_{c} \gtrsim 0.5\right)$ smooths the spectrum at all frequencies (fig. 11), preferentially scattering photons from low to high energy. As a consequence, the spectrum of the non-ionizing flux becomes steeper and the ionizing continuum hardens to an approximate power-law.

Because the width of the Lyman edge is dominated by relativistic boosting, inverse Compton scattering in an optically thick corona may reduce its amplitude but does not 
significantly broaden it (fig. 11). The amplitude of the emission feature reaches a minimum when $0.1 \lesssim \Theta \lesssim 1$. The actual value of $\Theta_{\min }$ depends upon the parameters of the corona and the input UV spectrum but the range of values is easily understood. Each input photon has a probability $\sim \tau_{c}$ of being scattered in the corona and the scattered photons are redistributed over a frequency range $\frac{\Delta \nu}{\nu} \sim \Theta$. Thus, very little redistribution occurs when $\Theta \ll 1$ and the scattered photons emerge at much higher energies when $\Theta \gg 1$.

The total optical depth of the corona is approximately the sum of the contributions from the soft X-ray corona (discussed in $\$ 4.1$.) and the hard X-ray corona, where the gravitational potential energy is released. In $\$ 4$.1. we demonstrated that a thermal runaway occurs for $\Sigma_{2} \sim 0.1 \mathrm{gm} \mathrm{cm}^{-2}$ for $r \lesssim 50$ and $\Sigma_{2} \sim 1 \mathrm{gm} \mathrm{cm}^{-2}$ at larger radii. This range of column densities corresponds to $\tau_{s x} \sim 0.04-0.4$. The Lyman edge is formed at $r \sim 10-20$, where $\tau_{s x} \lesssim 0.04$. The optical depth $\left(\tau_{h x}\right)$ and geometry of the hard X-ray corona are controversial, but may be estimated through the use of thermal Comptonization models. HM93 find $\tau_{h x} \lesssim 0.5$ assuming a uniform slab; Zdziarski et al. (1995) found $\tau_{h x} \simeq 0.08$ for the same geometry; Pietrini \& Krolik (1995) confirmed the Zdziarski et al. (1995) model, but also suggested that either clumping of the corona or a physical separation between it and the reprocessing surface would permit a match to the observed X-ray slope with larger $\tau_{h x}$. Stern, et al. (1995) prefer a model in which the corona is clumped, but close to the accretion disk, and has $\tau_{h x} \lesssim 0.1$.

If the corona is highly clumped, it can have little effect on the bulk of the UV photons. These modeling efforts indicate that a smooth corona must be rather optically thin, so it seems unlikely that the mean total Compton depth overlying the rings producing the Lyman edge can be any greater than a few tenths in most cases.

\section{Gravitational Instability of the Cold Disk}

Accretion disks are self-gravitating and unstable when (Toomre 1964, Goldreich \& Lynden-Bell 1965, Sakimoto \& Coroniti 1981)

$$
Q=\frac{z_{o} \Omega_{k}^{2}}{G \Sigma_{o}}<1
$$

or

$$
\rho_{o} \gtrsim \frac{M}{4 \pi R^{3}}=0.617 m_{8}^{-2} r^{-3} \mathrm{gm} \mathrm{cm}^{-3},
$$

where $\Omega_{k}$ is the Keplerian angular frequency. The range of length scales for the growing perturbations is $Q \lesssim l / z_{o} \lesssim 1 / Q$ (Toomre 1964). Comparing eqs. 14 and 47, we see that 
an isothermal accretion disk with no internal dissipation is gravitationally stable if

$$
\dot{m} m_{8} T_{5}^{-3 / 2} \alpha_{S S}^{-1} \lesssim 0.9
$$

The temperature of the isothermal disk is reasonably well approximated by the effective temperature of the irradiating flux (eq. A5) because the Thomson depth of the skin is near unity. In contrast, the central temperature of the standard optically thick disk can be more than an order of magnitude larger than $T_{\text {eff }}$. Inserting $T_{\text {eff }}$ into the stability criterion (eq. 48), we find that the isothermal disk is unstable at radii

$$
r \gtrsim 2 \dot{m}^{-5 / 9} m_{8}^{-11 / 9}
$$

The disk is self-gravitating at all radii $(r>3)$ when

$$
L \gtrsim 3 \times 10^{45} m_{8}^{-11 / 5} \operatorname{ergs~s}^{-1} .
$$

Of the disks we have considered, only the $\dot{m}=0.03$ and $m_{8}=0.27$ model satisfies this criterion over the entire range of radii. The case $\dot{m}=0.003$ and $m_{8}=2.7$ is stable for $r \lesssim 10$ and the remainder are unstable at all radii.

Although a strictly isothermal disk is unstable, a very small amount of internal dissipation will stabilize an irradiated disk. The gas pressure dominated solution for the disk structure described in SZ95 applies when there is enough internal dissipation (and optical depth) to significantly elevate the central temperature above $T_{\text {eff }}$ (SZ95 and appendix A.). This occurs when

$$
(1-f) \gg \frac{8}{3 \tau_{d}}(1-f / 2),
$$

where $\tau_{d}$ is the electron scattering optical depth of the disk and $f$ is the fraction of the gravitational potential energy which is dissipated in the corona (appendix A. and SZ95). As a result of the warmer internal temperature, the scale height increases and the density falls. We find that the central density drops below the critical density (eqs. 14 and 47) and the disk is stabilized when

$$
(1-f) \gtrsim 5 \times 10^{-16} \alpha_{S S}^{-7 / 3} m_{8}^{13 / 3} \dot{m}^{4 / 3} r^{9 / 2} .
$$

All of our models are stable at $r \lesssim 50$ with small values of $1-f$. The largest value of $1-f$ required for stability occurs in the $\dot{m}=0.03$ and $m_{8}=27$ case at $r=50$. Substantially smaller values of $1-f$ are needed to stabilize the disk at $r \lesssim 10$, where most of the Lyman edge radiation is produced, and for smaller values of $\dot{m}$ and $m_{8}$. In general, $4 \pi R^{3} \rho_{o} / M$ 
increases, and the value of $1-f$ required to stabilize the disk decreases, with increasing luminosity and $m_{8}$ (eq. 47).

From the scaling behavior we have already discovered, we may make some educated guesses about how the disk spectra will change if $1-f$ changes from zero to the small values required for stability. Because the density throughout the disk will fall, while X-ray heating will keep the gas temperature in the heated layer near $T_{\text {eff }}$, we expect the gas pressure to decrease with increasing $1-f$. Therefore, the ionization parameter in the heated skin will increase, and more of the incident X-ray flux will emerge as soft X-rays rather than in the ultraviolet. (\$4.1.). In addition, we expect the amplitude of the Lyman edge feature will increase as $1-f$ increases because of the lower gas pressure in the atmosphere (\$4.2.1.).

The disk is supported by radiation pressure at $r \sim 6$ when

$$
f \simeq 1-0.05 \dot{m}^{-8 / 9}\left(\alpha_{S S} m_{8}\right)^{-1 / 9}
$$

and over a larger range of radii for smaller $f$ (SZ95). Radiation pressure support will inflate the disk, suppressing the gravitational instability, but may cause the disk to become thermally unstable (e.g., Piran 1978). However, for our range of model parameters, the value of $f$ needed to stabilize the disk is much smaller than this value in all but the highest luminosity disk $\left(\dot{m}=0.3\right.$ and $\left.m_{8}=2.7\right)$.

\section{Conclusions}

We have calculated the vertical structure and UV spectrum of an accretion disk irradiated by hard X-rays from a corona, as predicted by many models for the X-ray emission in AGN (e.g., Pounds et al. 1990; HM93). Consistent with these X-ray production models, we assume there is no viscous dissipation in the accretion disk. The gravitational potential energy of the accreting gas is dissipated in a corona and radiated as hard X-rays with a power law spectrum. Photoionization of oxygen and iron by the coronal X-rays heat a geometrically thin skin at the surface of the accretion disk. Because there is no dissipation inside the disk proper, its temperature is constant as a function of altitude and the gas density and optical depth are much larger than in a conventional dissipative disk (SZ95).

To find the structure of the X-ray heated skin, we have solved the differential equations of radiative transfer, hydrostatic equilibrium and surface mass density subject to the constraints of radiative equilibrium and charge conservation. The inner boundary conditions for this numerical solution were chosen to match an analytical solution for the underlying 
isothermal accretion disk. We tested our method by computing the structure and spectrum of a conventional accretion disk and comparing to the previous state-of-the-art calculation (LN89, Sincell \& Krolik 1996).

The inner edge of the X-ray heated skin is 3-4 scale heights from the midplane of the isothermal disk. In the skin, but not the body of the disk, the force of the UV flux supports the gas against gravity and the momentum of the absorbed X-rays. The gas pressure in the skin is orders of magnitude smaller than the pressure at the midplane of the disk and is nearly constant throughout the skin. The gas temperature typically increases from the inner edge of the skin to the surface, although the temperature gradient occasionally changes sign. The changes in the temperature gradient correspond to changes in the ionization state and opacity of the gas.

The gas above the column density $\Sigma_{2}$ is unable to cool because cooling by thermal radiation processes is no longer able to balance heating by X-ray photoionization. This transition takes place at a characteristic value of the ionization parameter $\Xi \simeq 1$. Compton cooling was not included in this model so we could not find a solution for $\Sigma<\Sigma_{2}$. However, we estimate the gas temperature in the soft X-ray corona to be $T_{s x} \simeq 1.5 \times 10^{7} \mathrm{~K}$. We find that $\Sigma_{2}$ increases with disk radius, because the cooler gas at large radii radiates less efficiently, and the average value of $\Sigma_{2}$ for a given disk increases with the accretion rate, because $\Xi \propto \dot{m}$ at the surface of the heated skin.

For our specific X-ray spectrum and set of approximations, the fraction of the incident hard X-ray flux which is reprocessed into UV photons ranges from $f_{U V}=1.0$ at $\dot{m}=0.003$ to 0.62 when $\dot{m}=0.3$. The exact value of $f_{U V}$ depends on the shape of the X-ray spectrum, and our method (see §4.1) can be expected to slightly underestimate $f_{U V}$. Nevertheless, the decrease in $f_{U V}$ with accretion rate is a direct consequence of our assumption that all the gravitational potential energy is dissipated in the corona (see eq. 40) and should be a general property of irradiated disks.

The reprocessed UV continuum depends significantly on the specific parameters of the model (accretion rate, central mass), but certain features are generic: it has strong spectral curvature, in the sense that the spectrum softens with increasing frequency; and there is a significant Lyman edge emission feature. The low frequency continuum (parameterized by the spectral index at $10^{15} \mathrm{~Hz}$ ) hardens with increasing X-ray flux (i.e., $\dot{m} / m_{8}$ ) and ranges from -1.6 when $\dot{m} m_{8}^{-1} \sim 10^{-3}$ to 0.8 when $\dot{m} m_{8}^{-1} \sim 10^{-1}$. The continuum slope in this frequency range is only weakly dependent on viewing angle.

The Lyman edge is in emission because the photosphere is higher in the atmosphere at frequencies above the edge, and the temperature increases upward. Additional emission 
features at the HeI and HeII edges may also occur in the spectra of the hotter disks. The amplitude of the Lyman edge feature increases with central mass and is insensitive to the accretion rate. This is because both the photoionization and bremsstrahlung opacities are proportional to $P_{s} \propto m_{8}^{-1}$, so the gas cools less efficiently as $m_{8}$ increases. This results in larger temperature inversions which increase the strength of the emission feature. Doppler boosting spreads the Lyman edge over a range $\Delta \nu / \nu \sim \beta_{\phi} \sim 0.2$. The amplitude of the Lyman edge increases with disk inclination because the non-ionizing continuum is limb-darkened and the ionizing flux is (relatively) limb-brightened.

Irradiated disks are self-gravitating, and unstable, unless there is a small amount of internal dissipation. We find that the strictly isothermal disk is gravitationally unstable at $r \gtrsim 2 \dot{m}^{-5 / 9} m_{8}^{-11 / 9}$, so that strictly isothermal disks with luminosities $L \gtrsim 3 \times 10^{45} m_{8}^{-11 / 5} \mathrm{ergs} \mathrm{s}^{-1}$ are unstable at all radii. If, as is likely, there is a small amount of internal dissipation, and this stabilizes the disk, we expect $f_{U V}$ to decrease, and the amplitude of the Lyman edge feature to increase relative to what our models show for the limiting case of $f=1$. These effects are likely to become stronger for larger luminosity and central mass.

Some, but not all, of these predictions are consistent with observations. The average optical-UV power-law index for quasars is $\sim-0.5$ (Laor 1990) but a single power law is a poor description of the continuum spectrum (Francis, et al. 1991). The continuum of the composite quasar spectrum, formed by combining all the quasars in the Large Bright Quasar Survey, softens with increasing frequency (Francis, et al. 1991). Both the mean optical-UV slope and the spectral curvature are consistent with our predicted UV spectrum of an irradiated disk if $\dot{m} m_{8}^{-1} \sim 10^{-2}$.

On the other hand, our finding that a Lyman edge emission feature is a general property of X-ray-irradiated disks is difficult to reconcile with recent observations of AGN. Lyman edge features are rarely seen, and then usually as partial absorption edges (Antonucci, Kinney \& Ford 1989, Koratkar, Kinney \& Bohlin 1992). Emission features at the Lyman edge have been detected only in polarized light, and in these two quasars the total flux spectrum shows the Lyman edge in absorption (Koratkar, et al. 1995). The polarization of this emission is quite high and detailed polarization dependent calculations (e.g., Blaes \& Agol 1996) will be required to determine if these features could be produced by an irradiated disk.

Internal dissipation might, in some cases, reduce the amplitude of the Lyman edge feature. Conventional disks (Sincell \& Krolik 1996) sometimes produce emission features at the Lyman edge, and sometimes absorption, depending primarily on $\dot{m}$. However, both the fraction of the total gravitational energy dissipated in the disk and $\dot{m}$ must be very finely 
tuned to eliminate Lyman edge features from all AGN.

Inverse Compton scattering in the disk coronae can smooth out the Lyman edge feature only if the hot corona producing the hard X-rays covers most of the disk and has an optical depth $\tau_{c} \gtrsim 0.5$. Because most recent treatments of X-ray production suggest that either the optical depth is rather smaller than this, or else the hot corona is highly clumped, it is unlikely that Compton scattering smooths the Lyman edge.

The only escape we see from this contradiction makes use of the fact that the evidence for X-ray irradiation comes almost entirely from low luminosity AGN (i.e., Seyfert galaxies), while spectra of the Lyman edge region exist only for high luminosity AGN (i.e., quasars). The reason for this separation is that only the Seyfert galaxies, which are relatively nearby, are bright enough for detailed X-ray spectroscopy, while in order for us to see the Lyman edge, we must look at objects with redshifts great enough to bring it below the Lyman cut-off imposed by our Galaxy's interstellar medium. Perhaps, then, Seyfert galaxies have Lyman edges in emission (if we could only see them), while quasar disks are not so thoroughly irradiated by X-rays. X-ray spectroscopy of quasars by future, more sensitive instruments, will be able to test this idea.

Thus, our calculation of the UV spectrum expected from irradiated disks raises serious questions for accretion disk models. While the X-ray production and X-ray reflection models which motivated this study have had substantial success in describing AGN X-ray spectra, we now see that they also predict strong Lyman edge emission features, a prediction that is contradicted by the simplest interpretation of the observations.

We would like to thank Ari Laor for helpful conversations and for providing many disk spectra. We also thank Omer Blaes and Eric Agol for ongoing conversations. MWS also thanks the Observatoire de Meudon for hospitality during part of this work. MWS received support for this work from NASA grants NAGW-3129, 1583 and NAG 5-2925, and NSF grant AST 93-15133. JHK was partially supported by NASA Grant NAGW-3156.

\section{REFERENCES}

Antonucci, R. R. J., A. L. Kinney \& H. C. Ford 1989 Ap. J., 342, 64

Auer, L. H. \& D. Mihalas 1970 M.N.R.A.S., 149, 65

Bell, K. L. \& A. E. Kingston 1967 Proc. Phys. Soc., 90, 31

Begelman, M. C., C. F. McKee \& G. A. Shields 1983 Ap. J., 271, 70 
Blaes, O. M. \& E. Agol, 1996, submitted to Ap. J. Letters

Clavel, J., et al. 1992 Ap. J., 393, 113

Collin-Souffrin, S. 1991 Ast. Ap., 249, 344

Cunningham, C. T. 1975 Ap. J., 202, 788

Daltabuit, E. \& D. P. Cox 1972 Ap. J., 177, 855

Ferland, G. J. \& M. J. Rees 1988 Ap. J., 332, 141

Francis, P. J., et al. 1991 Ap. J., 373, 465

Ghisellini, G., Haardt, F., \& Matt, G. 1994 M.N.R.A.S., 267, 743

Goldreich, P. \& D. Lynden-Bell 1965 M.N.R.A.S., 130, 7

Green, P.J., Schartel, N., Anderson, S.F., Hewett, P.C., Foltz, C.B., Brinkmann, W., Fink, H., Trümper, J., and Margon, B. 1995 Ap. J., 450, 51

Guilbert, P. W. \& M. J. Rees 1988 M.N.R.A.S., 233, 475

Haardt, F. \& L. Maraschi 1993 Ap. J., 413, 507 (HM93)

Haardt, F. 1993 Ap. J., 413, 680

Haardt, F., L. Maraschi \& G. Ghisellini 1994 Ap. J., 432, L95

Kolykhalov, P.I. and Sunyaev, R.A. 1984 Adv. Sp. Res., 3,249

Koratkar, A., A. L. Kinney \& R. C. Bohlin 1992 Ap. J., 400, 435

Koratkar, A., R. R. J. Antonucci, R. W. Goodrich, H. Bushouse \& A. L. Kinney 1995 Ap. J., 450, 501

Krolik, J.H., Madau, P. \& Życki, P. 1994 Ap. J., 420, L57

Krolik, J. H., C. F. McKee \& C. B. Tarter 1981 Ap. J., 249, 422

Krolik, J. H., et al. 1991 Ap. J., 371, 541

Laor, A. \& H. Netzer 1989 M.N.R.A.S., 238, 897 (LN89)

Laor, A., H. Netzer \& T. Piran 1990 M.N.R.A.S., 242, 560

Laor, A. 1990 M.N.R.A.S., 246, 369

Laor, A. 1992, in Testing the AGN Paradigm: AIP Conference Proceedings 254, American Institute of Physics, New York

Lightman, A. P. \& T. R. White 1988 Ap. J., 335, 57

Lin, D. N. C., \& J. E. Pringle 1987 M.N.R.A.S., 225, 607

London, R., R. McCrae \& L. H. Auer 1981 Ap. J., 243, 970 
Malkan, M. A. \& W. L. W. Sargent 1982 Ap. J., 254, 22

Malkan, M. A. 1983 Ap. J., 268, 582

Martocchia, A. and Matt, G. 1996, M.N.R.A.S. in press

Mihalas, D. M., 1978, Stellar Atmospheres, W. H. Freeman \& Co., San Francisco

Nandra, K. 1997, in IAU Coll. 159, Emission Lines in Active Galactic Nuclei: New Methods and Techniques, eds. B. Peterson, F.-Z. Cheng, and A.S. Wilson

Nandra, K. \& K. A. Pounds 1994 M.N.R.A.S., 268, 405

Novikov, I. \& K. S. Thorne, 1973 in Black Holes, p. 422, eds: deWitt, C. \& B. deWitt, Gordon \& Breach, New York

Page, D. N. \& K. S. Thorne 1974 Ap. J., 191, 499

Perola, G.C., et al. 1986 Ap. J., 306, 508

Peterson, B. M., et al. 1991 Ap. J., 368, 119

Pietrini, P. \& J. H. Krolik 1995 Ap. J., 447, 526

Piran, T. 1978 Ap. J., 221, 652

Pounds, K. A. \& T. J. Turner, 1988 Proceedings of the IAU Symposium 134 "Active Galactic Nuclei", eds. J. S. Miller \& D. E. Osterbrock, Kluwer, Dordrecht

Press, W. H., S. A. Teukolsky, W. T. Vetterling \& B. P. Flannery, 1992, Numerical Recipes in C, Cambridge University Press

Riffert, H. \& H. Herrold 1995 Ap. J., 450, 508

Rokaki, E., Collin-Souffrin, S., \& Magnan, C. 1993 Ast. Ap., 272, 8

Ross, R. R. \& A. C. Fabian 1993 M.N.R.A.S., 261, 74

Rybicki, G. B. 1971 J. Quant. Spec. Rad. Transfer, 11,589

Rybicki, G. B. \& A. P. Lightman, 1979, Radiative Processes in Astrophysics, John Wiley \& Sons, Inc., New York

Sakimoto, P. J. \& F. V. Coroniti 1981 Ap. J., 247, 19

Shakura, N. I. \& R. A. Sunyaev 1973 Ast. Ap., 24, 337 (SS73)

Shakura, N. I. \& R. A. Sunyaev 1976 M.N.R.A.S., 175, 613

Shields, G. A. 1978 Nature, 272,706

Sincell, M. W. \& J. H. Krolik, 1996, in preparation

Stern, B. E., J. Poutanen, R. Svensson, M. Sikora \& M. C. Begelman 1995 Ap. J., 449, L13 
Stocke, J., Morris, S.L., Gioia, I.M., Maccacaro, T., and Schild, R. 1991 Ap. J. (Supp.)., 76,813

Sun, W.-H. and Malkan, M.A. 1989 Ap. J., 346, 68

Toomre, A. 1964 Ap. J., 139, 1217

Svensson, R. \& A. A. Zdziarski 1995 Ap. J., 436, 599 (SZ95)

Voit, G.M. \& Shull, J.M. 1988 Ap. J., 331, 197

Walter, R. \& H. H. Fink 1993 Ast. Ap., 274, 105

Wamsteker, W., et al. 1990 Ap. J., 354, 446

Wilkes, B.J., Tananbaum, H., Worrall, D.M., Avni, Y., Oey, M.S., and Flanagan, J. 1994 Ap. J. (Supp.)., 92,53

Zdziarski, A.A., Johnson, W.N., Done, C., Smith, D. \& McNaron-Brown, K. 1995 Ap. J., 438, L63

Zheng, W., Kriss, G.A., Telfer, R.C., Grimes, J.P., and Davidsen, A.F. 1996, Ap.J. in press Życki, P. T., J. H. Krolik, A. A. Zdziarski \& T. R. Kallman 1994 Ap. J., 437, 597

\section{A. Radial Structure of the Cold Disk for $f \simeq 1$}

The radial structure equations for the cold gas pressure dominated disk are (SZ95)

$$
\begin{gathered}
P=\frac{G M \rho}{2 R^{3}} z_{o}^{2}=\frac{\rho c^{2}}{4} r^{-1}\left(\frac{z_{o}}{R}\right)^{2} \\
P=\frac{\dot{M} \omega}{4 \pi \alpha_{S S}} \frac{\sqrt{\pi}}{z_{o}}=\sqrt{\frac{\pi}{8}} \frac{m_{p} c^{2}}{\sigma_{T} R_{s}} r^{-5 / 2} \frac{\dot{m}}{\alpha_{S S} \eta}\left(\frac{z_{o}}{R}\right)^{-1}
\end{gathered}
$$

and the perfect gas equation of state. The gas temperature of the disk is determined by the X-ray heating rate (eq. 25)

$$
T \simeq\left(\frac{(1-f / 2) Q_{d i s}}{\sigma}\right)^{1 / 4}
$$

when $f \simeq 1$. SZ95 assume that the gas temperature is proportional to the effective temperature of the flux dissipated internally and neglect the X-ray heating. Consequently, the SZ95 solution for the radial structure is singular $(\rho \rightarrow \infty, T=0)$ when $f=1$. 
The solution for the radial structure is straightforward. We find that the scale height is

$$
z_{o}^{2}=\frac{2 k T_{e f f} R^{3}}{G M m_{p}}(1-f / 2)^{1 / 4},
$$

where

$$
T_{e f f}=\left(\frac{Q_{d i s}}{\sigma}\right)^{1 / 4}
$$

The midplane gas density is

$$
\rho=0.53 \dot{m} m_{8}^{-1} \alpha^{-1} r^{-3} T_{e f f}^{-3 / 2}(1-f / 2)^{-3 / 8}
$$

where we have inserted the scalings in eq. 9. These equations reduce to the isothermal disk solution (eqs. 7 and 14) for a disk temperature of $T_{g}=T_{\text {eff }}$ when $f=1$, as expected.

The introduction of a new definition of the disk temperature removes the singularity of the SZ95 solution at $f=1$. The cold disk temperature will never fall to zero, as predicted by SZ95, because of the X-ray heating by the corona. As a result, the scale height is non-zero and the density remains finite for $f=1$. In addition, we find that the scale height and midplane density of a gas pressure dominated disk are insensitive to the actual value of $f$, so long as it is close enough to unity that the central temperature is determined primarily by irradiation ( $c f . \S 5)$. 


\section{Figure and Table Captions}

Figure 1. The X-ray heating rate and integrated UV flux as a function of column density. Units are arbitrary.

Figure 2. The structure of the X-ray heated skin at $r=14.0$ for $\dot{m}=0.03$ and $m_{8}=2.7$. The panels show the vertical coordinate scaled to the value at the inner edge of the skin $\left(Z_{s}\right)(\mathrm{a})$, the gas pressure (b), the gas temperature (c) and the location of the photosphere $(d)$.

Figure 3. The mass column of the thermal runaway $\left(\Sigma_{2}\right)$ for fixed $\dot{m}$ and variable $m_{8}$. The outer boundary of the integration is $\Sigma=10^{-4} \mathrm{~g} \mathrm{~cm}^{-2}$.

Figure 4. The mass column of the thermal runaway $\left(\Sigma_{2}\right)$ for fixed $m_{8}$ and variable $\dot{m}$. The outer boundary of the integration is $\Sigma=10^{-4} \mathrm{~g} \mathrm{~cm}^{-2}$.

Figure 5. The fractional abundances of HeII and HeIII at $\Sigma_{2}$ for $\dot{m}=0.3$ and $m_{8}=2.7$. The points are $\Sigma_{2}$ for this model.

Figure 6. The spectrum at $r=14.0$ for $\dot{m}=0.03$ and $m_{8}=2.7$. The crosses are the results of the simulation and the dashed line represents a black body spectrum at the effective temperature of the disk.

Figure 7. The face-on disk spectrum for a fixed mass accretion rate and varying central mass.

Figure 8. The face-on disk spectrum for a fixed central mass and varying mass accretion rate.

Figure 9. The disk spectrum as a function of inclination, $\mu=\cos \theta$.

Figure 10. The Comptonized UV spectrum of a face-on disk for a fixed $\tau_{c}$ and varying $T_{c}$. The disk parameters are $\dot{m}=0.03$ and $m_{8}=2.7$.

Figure 11. The Comptonized UV spectrum of a face-on disk for a fixed $T_{c}$ and varying $\tau_{c}$. The disk parameters are $\dot{m}=0.03$ and $m_{8}=2.7$. 
Table 1. The fraction of the incident X-ray flux which is reprocessed into thermal UV emission $\left(f_{U V}\right)$ and the continuum spectral index $\left(\alpha_{15}\right)$ for different models. 


\begin{tabular}{||c|c|c|c|c||}
\hline$\dot{m}$ & $m_{8}$ & $L_{T}$ & $f_{U V}$ & $\alpha_{15}$ \\
\hline 0.003 & 2.7 & $10^{44}$ & 1.0 & -1.2 \\
0.03 & 0.27 & $10^{44}$ & 0.96 & 0.8 \\
0.03 & 2.7 & $10^{45}$ & 0.91 & -0.2 \\
0.03 & 27.0 & $10^{46}$ & 0.85 & -1.6 \\
0.3 & 2.7 & $10^{46}$ & 0.62 & 0.3 \\
\hline
\end{tabular}

Table 1: 

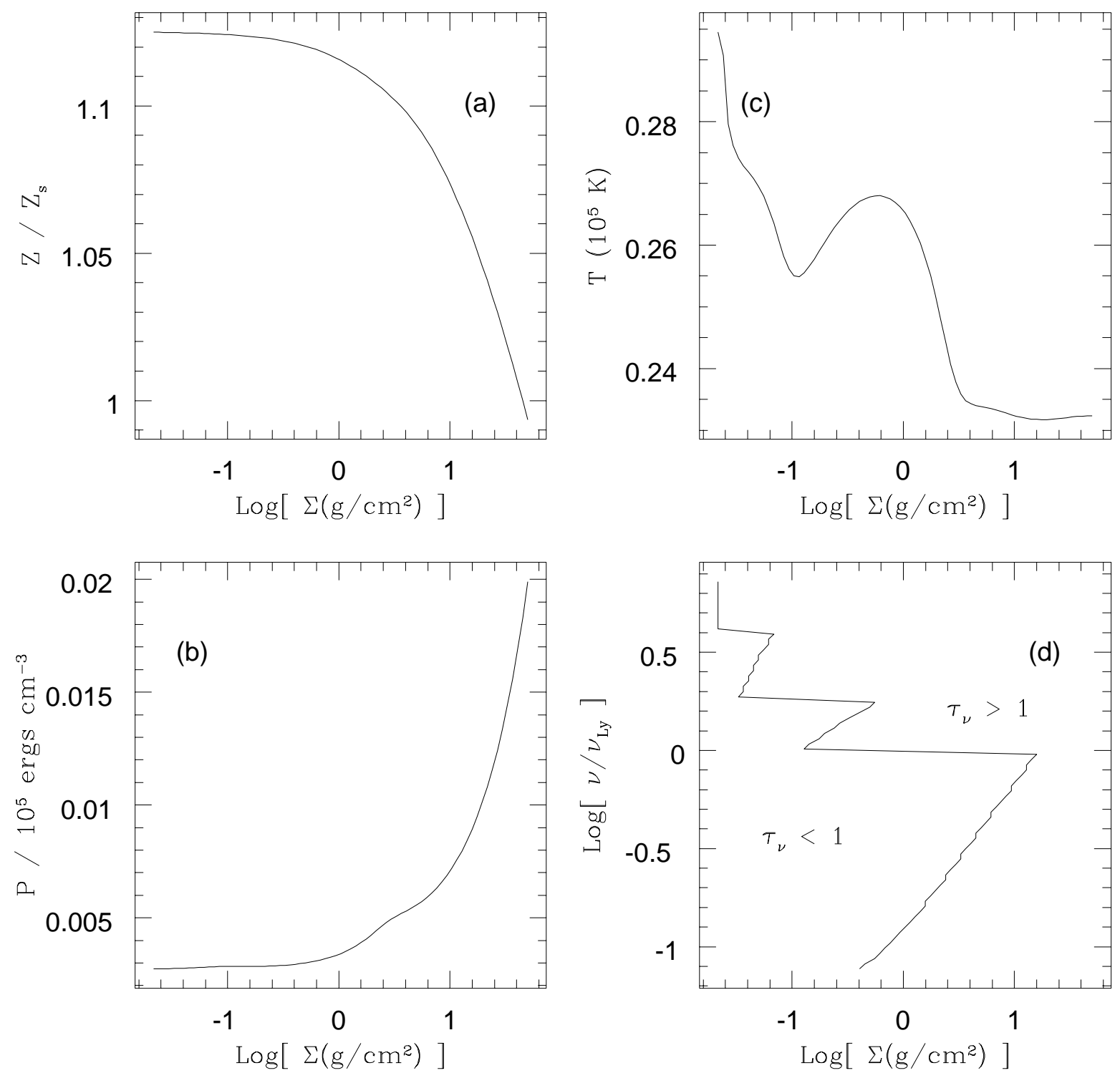

Fig. 1.- 

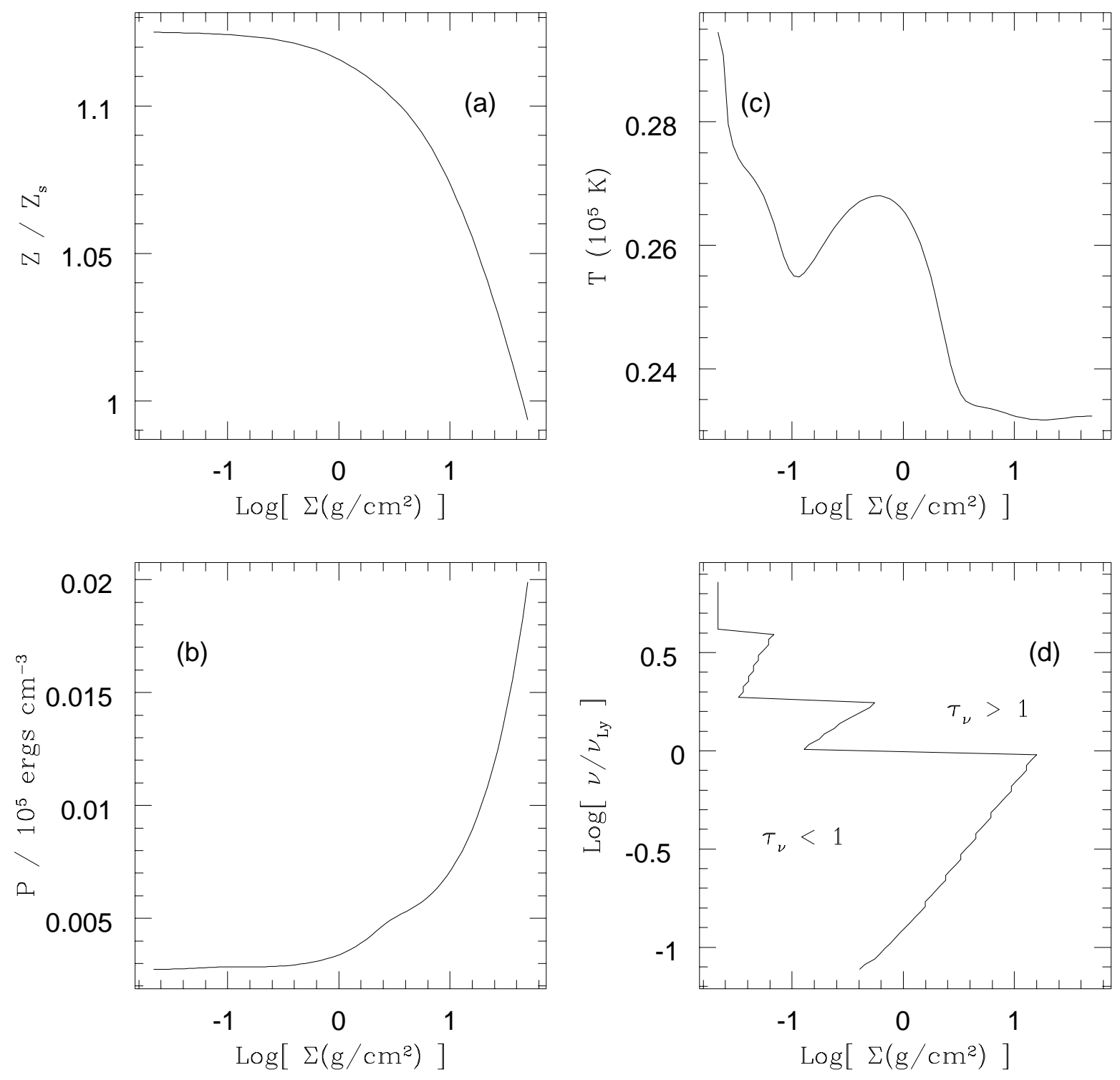

Fig. 2.- 


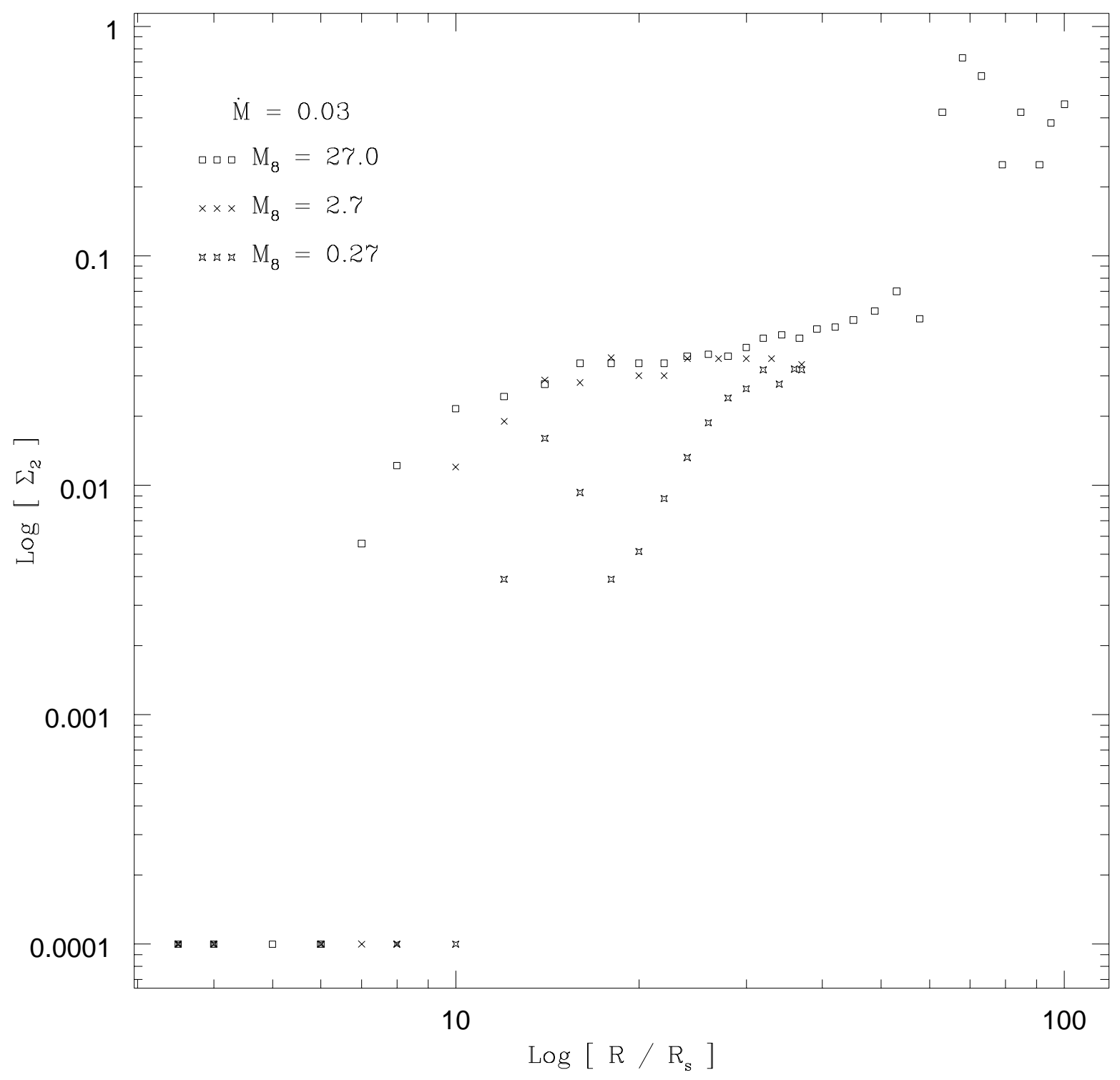

Fig. 3.- 


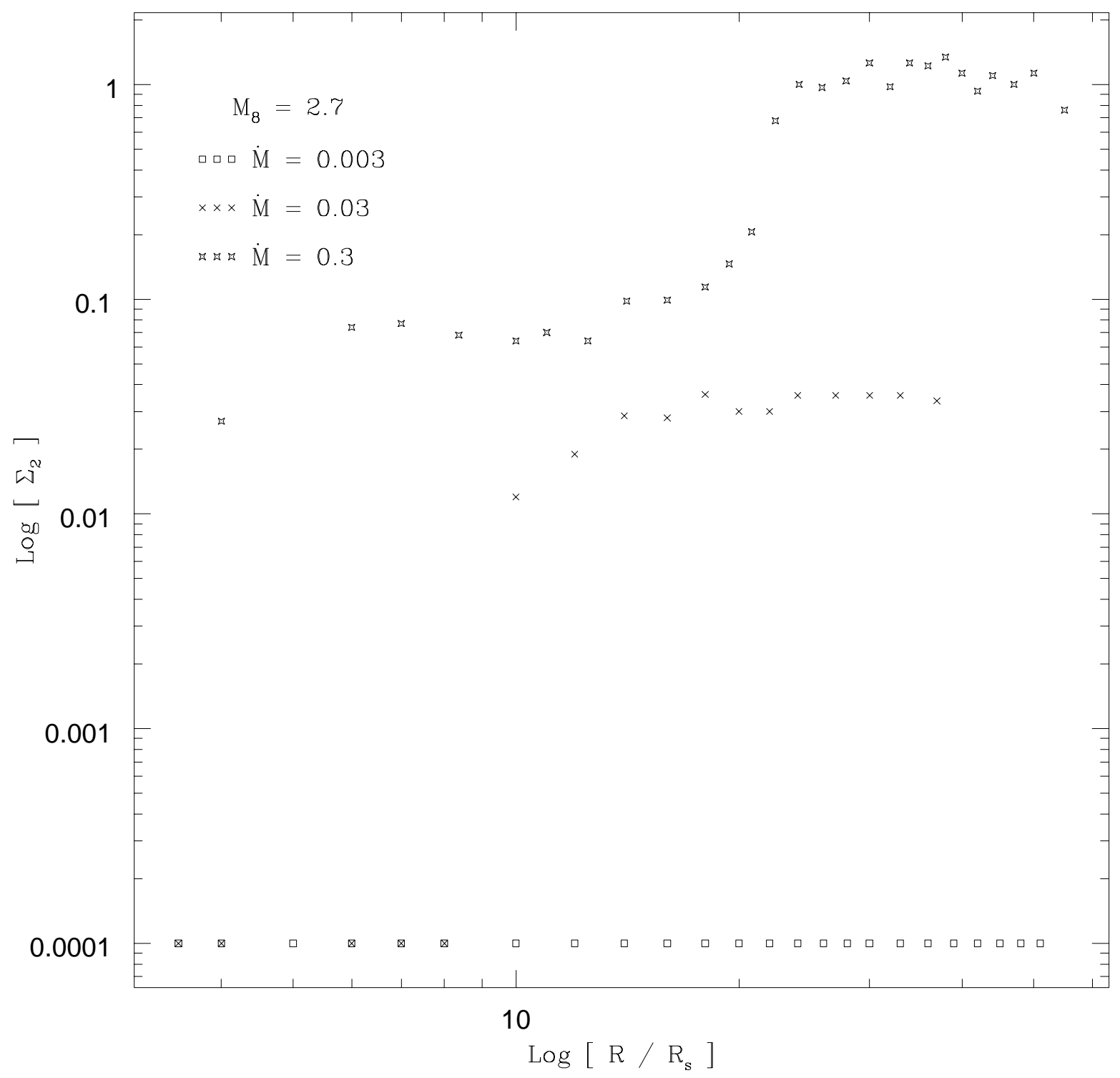

Fig. 4.- 


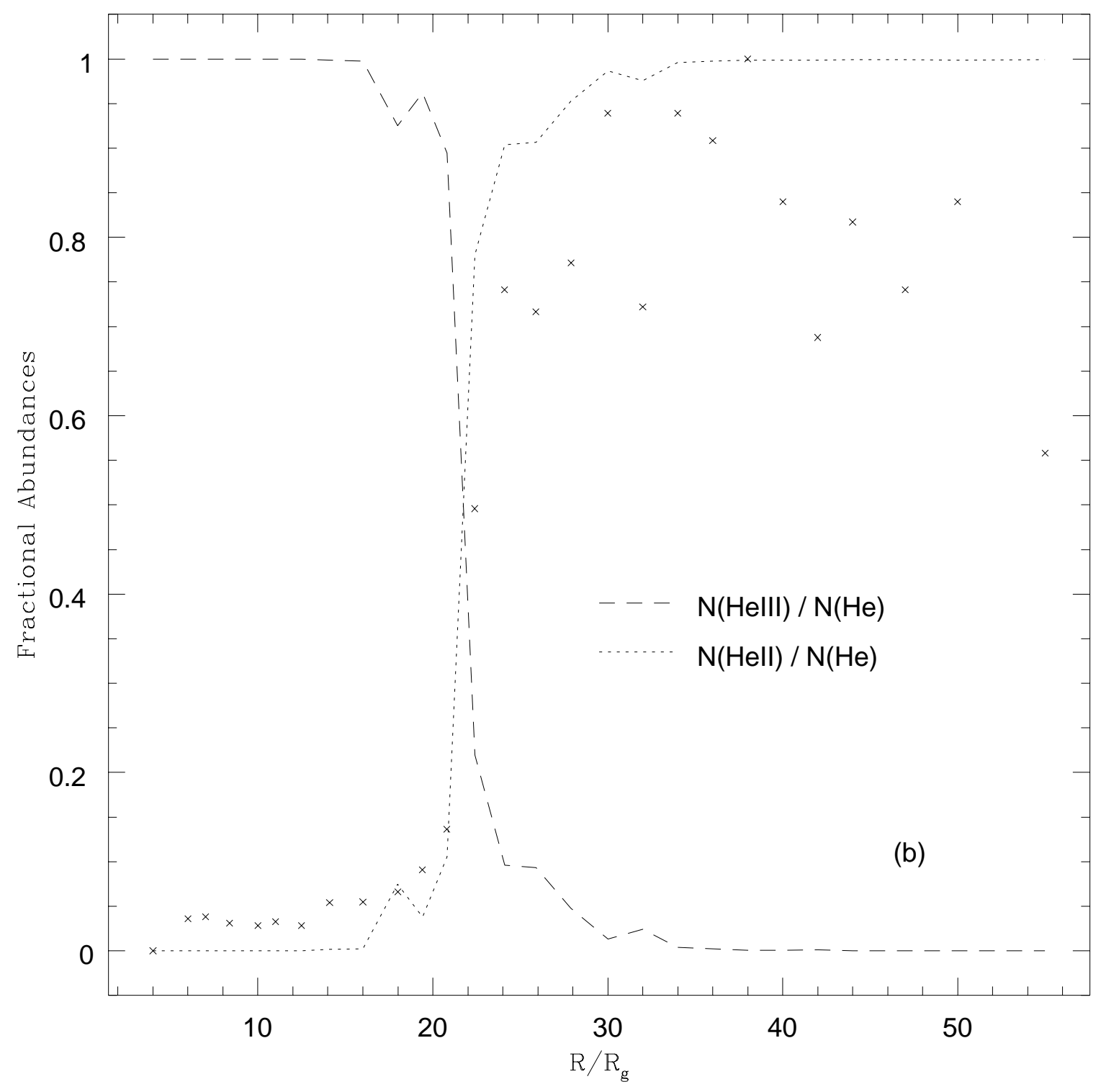

Fig. 5.- 


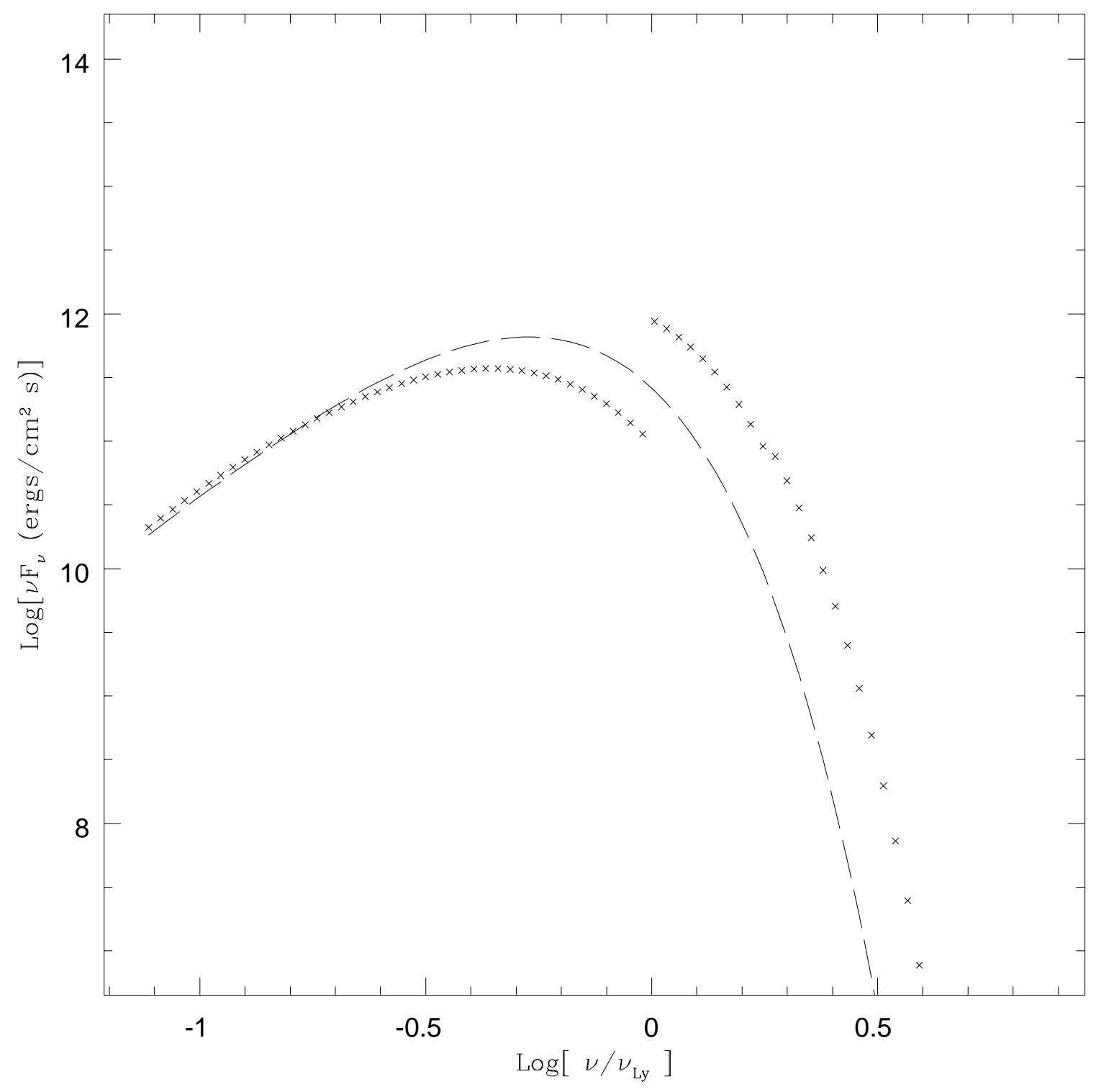

Fig. 6.- 


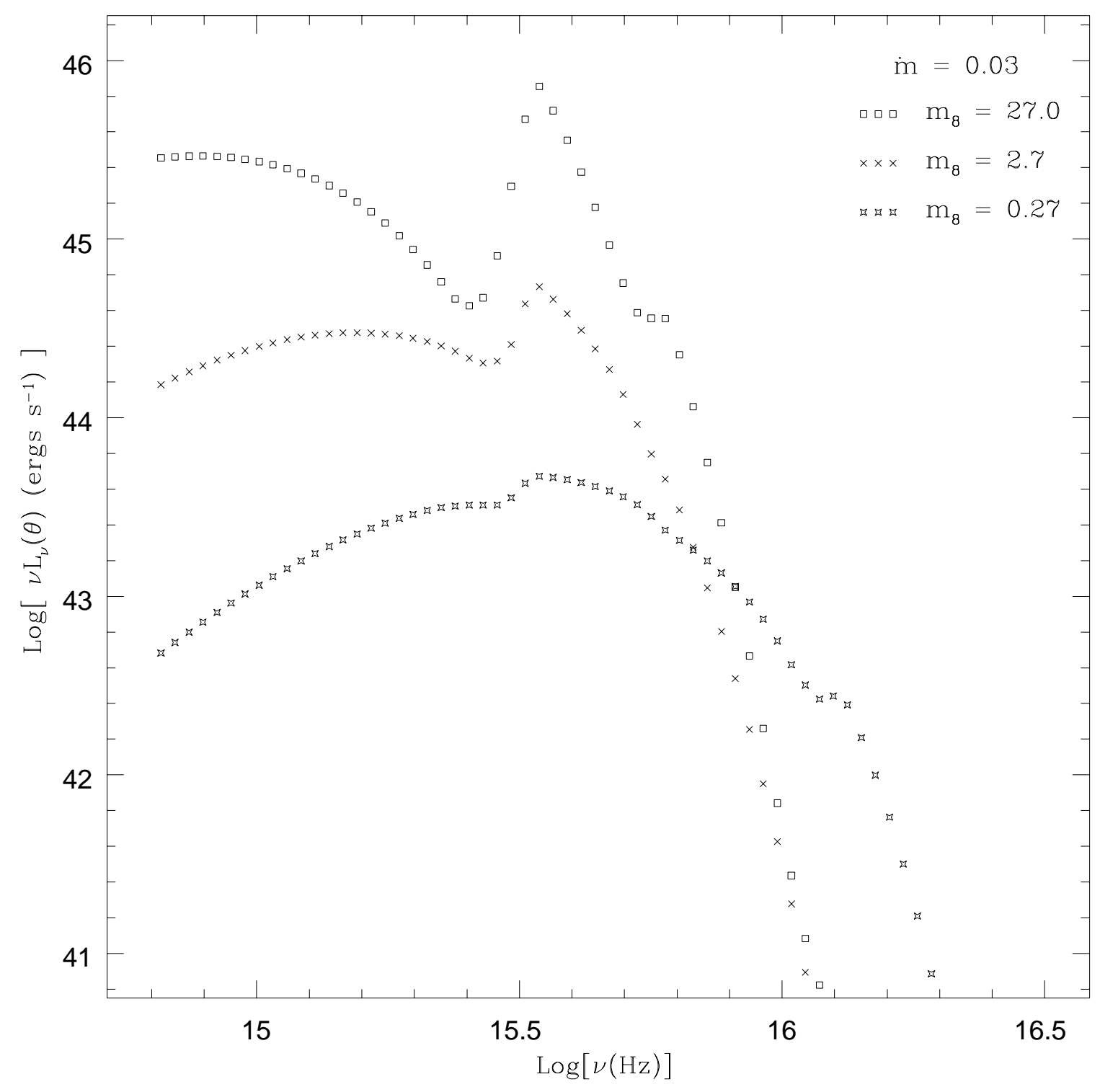

Fig. 7.- 


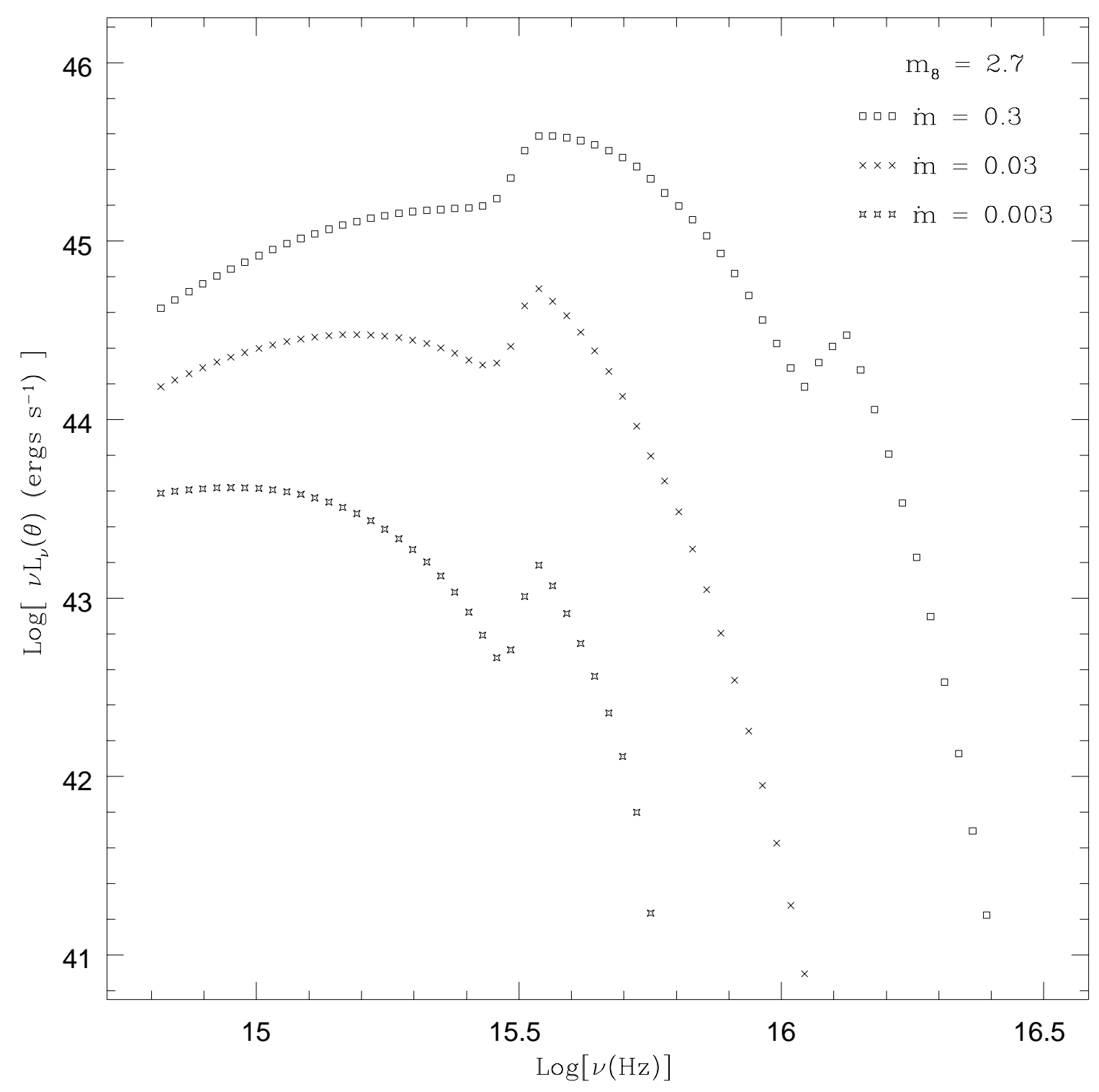

Fig. 8.- 


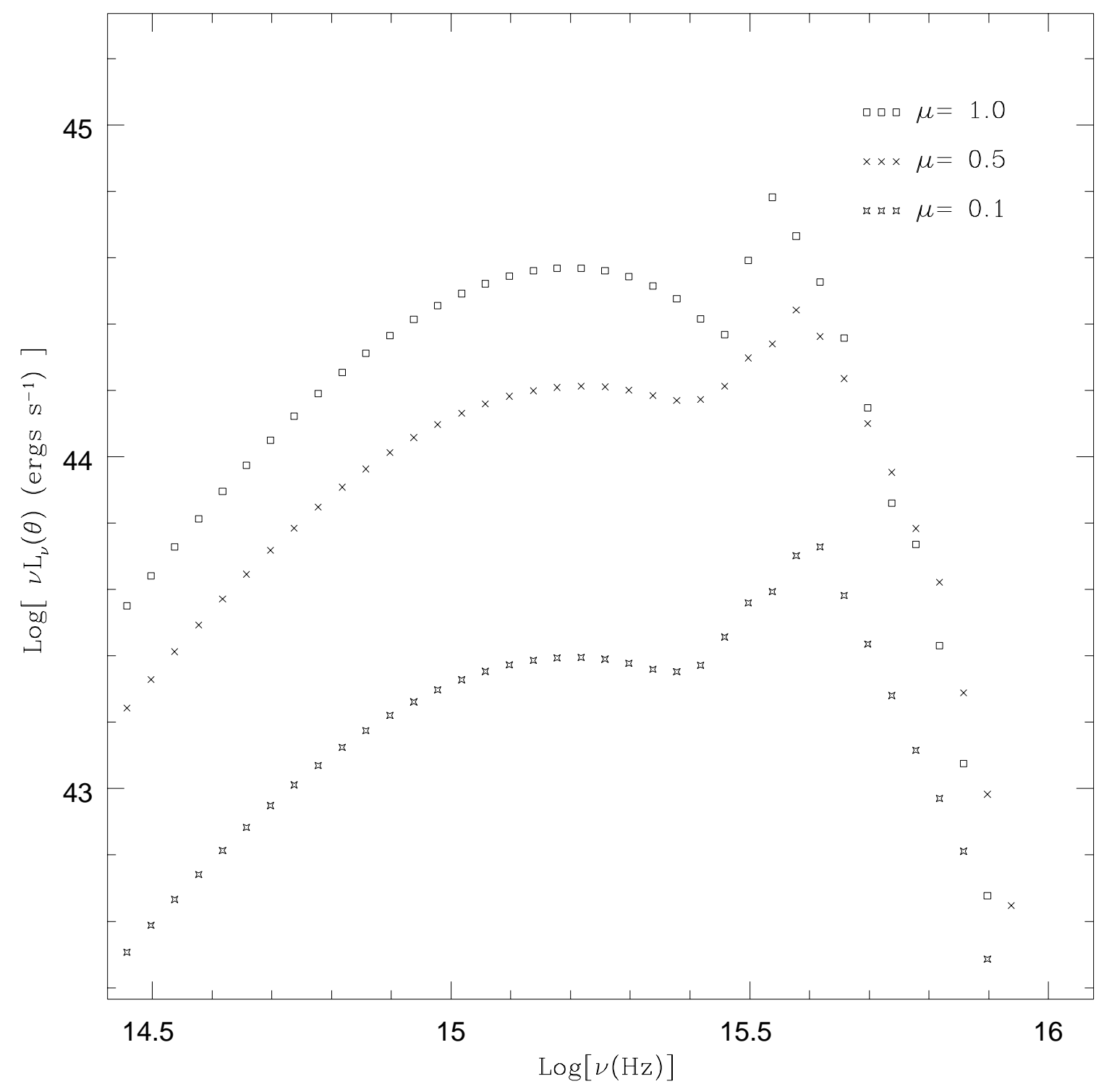

Fig. 9.- 


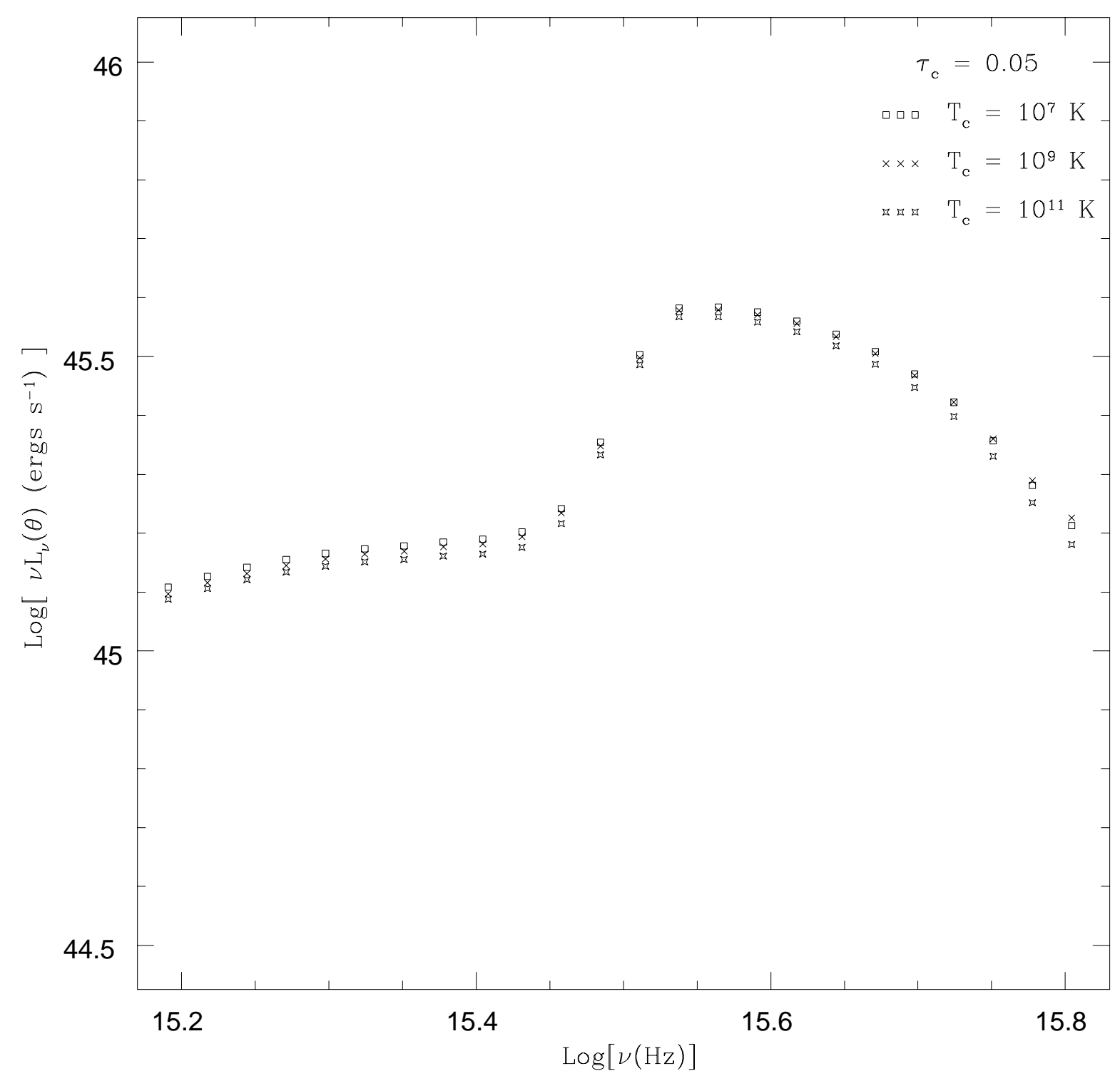

Fig. 10.- 


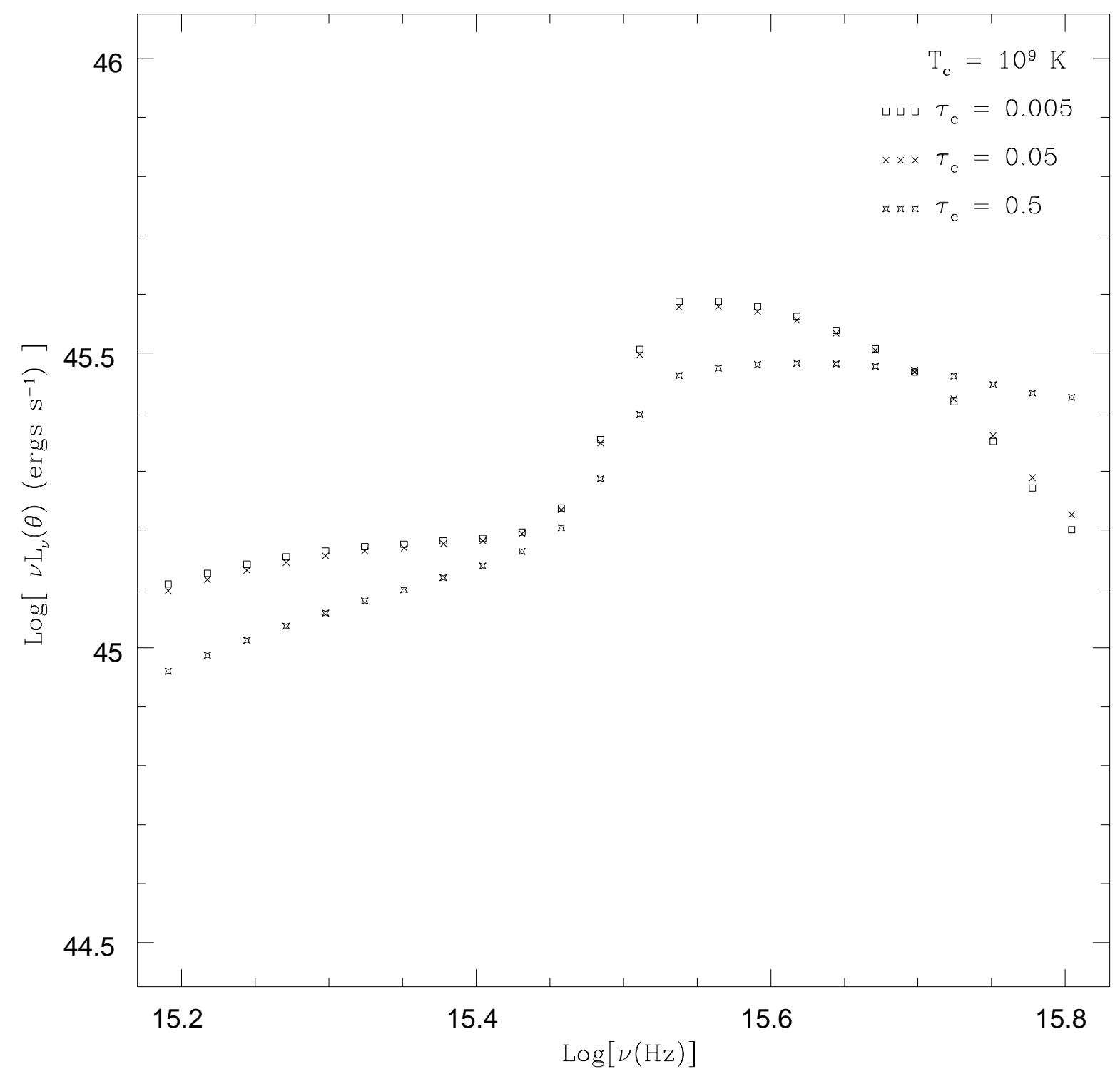

Fig. 11.- 